Research article

urn:1sid:zoobank.org:pub:09F625AD-D58F-4D26-98B7-832743127667

\title{
Contribution to the genus Chilocorus Leach, 1815 (Coleoptera: Coccinellidae: Chilocorini), with descriptions of two new species from China
}

\author{
Wenjing $\mathrm{LI}^{1}$, Lizhi $\mathrm{HUO}^{2}$, Di WANG ${ }^{3}$, Dirk AHRENS ${ }^{4} \&$ Xingmin WANG ${ }^{5, *}$ \\ 1,2,5 Key Laboratory of Bio-Pesticide Innovation and Application, Engineering Technology Research \\ Center of Agricultural Pest Biocontrol, Guangdong Province, Department of Entomology, South China \\ Agricultural University, Guangzhou 510640, China. \\ ${ }^{1}$ Plant Protection Research Institute, Guangdong Academy of Agricultural Sciences, \\ Guangzhou, Guangdong 510640, China. \\ ${ }^{3}$ People's Public Security University of China, Beijing 100038, China. \\ ${ }^{4}$ Zoologisches Forschungsmuseum Alexander Koenig Bonn, Adenauerallee 160, \\ 53113 Bonn, Germany. \\ *Corresponding author: wangxmen@scau.edu.cn \\ ${ }^{1}$ Email: 1wj4631221@aliyun.com \\ 2Email: huolizhicn@163.com \\ ${ }^{3}$ Email: jgxxywjywd@163.com \\ [4Email: d.ahrens@zfmk.de

\footnotetext{
${ }^{1}$ urn:1sid:zoobank.org:author:6B58B81E-DF8E-4463-8008-3CA190F588DD

${ }^{2}$ urn:Isid:zoobank.org:author:FA135086-13FD-4DF0-B5F9-8E3EE2111EF7

${ }^{3}$ urn:1sid:zoobank.org:author:7ED9BC5C-2112-483C-A39B-50259D5AB7CB

${ }^{5}$ urn:1sid:zoobank.org:author:51CDA9D0-12F3-4593-A887-BF8CB2E6AB16
} \\ ${ }^{4}$ urn:lsid:zoobank.org:author:DEDCE5CF-AA11-4BBF-A2C6-D7C815019714
}

\begin{abstract}
Twenty species of the genus Chilocorus Leach, 1815 currently known from China are recorded, including two new species described here: C. nigricaeruleus Li \& Wang sp. nov. and C. strenotubus Li \& Wang sp. nov. Diagnoses and detailed descriptions of the new species are given. Each species is illustrated in detail, including genitalia. Distribution maps, a key to the Chinese species and a world checklist of Chilocorus are given.
\end{abstract}

Keywords. Lady beetles, Chilocorini, new species, China, world checklist.

Li W., Huo L., Wang D., Ahrens D. \& Wang X. 2018. Contribution to the genus Chilocorus Leach, 1815 (Coleoptera: Coccinellidae: Chilocorini), with descriptions of two new species from China. European Journal of Taxonomy 469: 1-34. https://doi.org/10.5852/ejt.2018.469

\section{Introduction}

The tribe Chilocorini Mulsant, 1846 belongs to the subfamily Chilocorinae along with the tribes Platynaspini Mulsant, 1846 and Telsimiini Casey, 1899 in the traditional classification, based on 
the fact that they share a characteristic head capsule with a strongly expanded clypeus and reduced antennae (Sasaji 1968b). However, recent studies on the molecular phylogeny of Coccinellidae indicate that this subfamily is not a monophyletic group (Giorgi et al. 2009; Seago et al. 2011). As per the classification proposed by Ślipiński (2007) and Seago et al. (2011), Chilocorinae and its three tribes are now included in the subfamily Coccinellinae. Chilocorini has been identified as a sister group of Coccinellini (Magro et al. 2010; Seago et al. 2011; Escalona et al. 2017; Chen et al. 2017). At present, Chilocorini contains 27 genera and more than 280 species (Łączyński \& Tomaszewska 2012; Li et al. 2017).

Chilocorus, the largest genus of the tribe Chilocorini, contains 79 species distributed all over the world and mainly preying on coccoidea (Giorgi et al. 2009; Escalona et al. 2017). Many species of Chilocorus are economically important as they are widely used as biological control agents (Ślipiński \& Giorgi 2006), such as Chilocorus nigrita (Fabricius, 1798), which is widely distributed due to its deliberate introduction for biological control (Booth 1998). Leach (1815) established the genus Chilocorus for Coccinella cacti Linnaeus, 1767. Sicard (1920) proposed a subgenus C. (Trichocorus) for two species, this was supported by Chapin (1965) who considered Chilocorus as a non-monophyletic genus. This concept was accepted by Miyatake (1970), who studied East-Asian species, and subdivided Chilocorus into seven groups depending on the characters of pronotal oblique line, prosternal process, prosternal hypomeral foveae, elytral epipleural foveae, elytral outer margin, etc. The phylogenetic relationships of Chilocorus are still unclear. There are no detailed phylogenetic analyses based on morphology and Chilocorini are poorly represented in the molecular studies dealing with Coccinellidae.

The species of Chilocorus are abundant in China and prior to the present study, 18 were known (Liu 1963; Pang \& Mao 1979; Cao \& Xiao 1984; Pang et al. 2004; Ren et al. 2009). However, no comprehensive revision of Chinese Chilocorus has been carried out over the last 40 years. Furthermore, the world checklist of Chilocorus has not been updated for several decades since Korschefsky (1932), who provided the world checklist of Coccinellidae. The present paper aims at updating the knowledge of Chinese Chilocorus, including two new species, and provides a checklist of the known species of the world.

\section{Material and methods}

Specimens examined in this study were collected from China and Nepal. Type specimens of the new species are deposited at the Department of Entomology, South China Agriculture University, Guangzhou. Other material examined is deposited in the following research institutions:

$\mathrm{SCAU}=$ South China Agriculture University, Guangzhou, China

FIQB $=$ The Forest Inspection and Quarantine Bureau, Yunnan, China

$\mathrm{NCHU}=$ National Chung Hsing University, Taiwan, China

External morphology was observed with a dissecting stereo microscope (SteREO Discovery V20, Zeiss). Male and female genitalia were dissected, cleared in a $10 \%$ solution of $\mathrm{NaOH}$ by boiling for several minutes and examined with an Olympus BX51 microscope. The photographs of genitalia and other morphological characters were generated with digital cameras (AxioCam HRc and Coolsnap-Procf \& CRI Micro*Color), attached to microscopes using AxioVision Rel. ver. 4.8 and Image-Pro Plus ver. 6.0 to capture images, and photographs were cleaned up and laid out in plates with Adobe Photoshop CS ver. 8.0. Terminology follows Ślipiński (2007) and Ślipiński \& Tomaszewska (2010).

\footnotetext{
Abbreviations

$\mathrm{TL}=$ total length, length from apical margin of clypeus to apex of elytra

$\mathrm{TW}=$ total width, width across both elytra at widest part

$\mathrm{TH}=$ height measured across the highest point of the elytra
} 


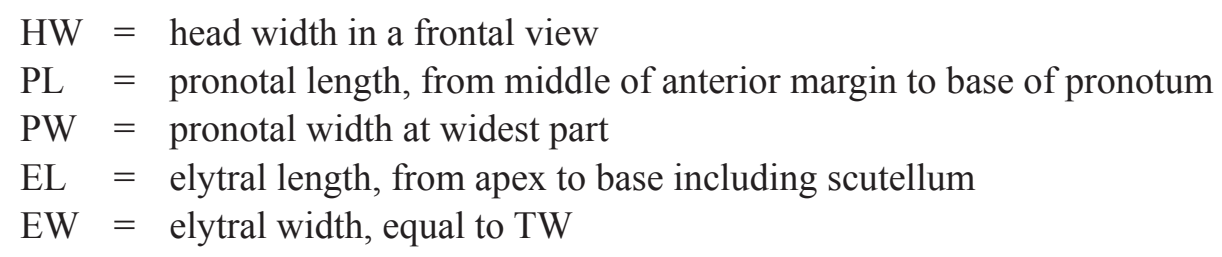

\title{
Results
}

\author{
Superfamily Coccinelloidea Latreille, 1807 \\ Family Coccinellidae Latreille, 1807 \\ Subfamily Coccinellinae Latreille, 1807 \\ Tribe Chilocorini Mulsant, 1846
}

Genus Chilocorus Leach, 1815

Chilocorus Leach, 1815: 116.

\section{Type species}

Coccinella cacti Linnaeus, 1767, by monotypy.

\section{Diagnosis}

The genus Chilocorus can be distinguished from the other genera of the tribe Chilocorini by the following characters: body with dorsum glabrous, rarely with pubescence; outer elytral margin slightly reflexed, without distinct bead; antenna stout, composed of 8 antennomeres (Fig. 3e); terminal maxillary palpomere elongate, from 1 to 2 times as long as basal width, with sides nearly parallel or moderately expanded to apex (Fig. 3f); prosternal process long, narrow and subparallel without carina; legs with stout femora, tibiae with a triangular tooth at basal $1 \frac{1}{3}$, without tibial spurs (Fig. 3i-j); tarsal claws stout, with approximately rectangular basal tooth, about $1 \frac{1}{2}$ length of claw (Fig. 3k).

\section{Description}

BoDy. Broadly oval or roundish, hemispherical and strongly convex. Dorsum glabrous, rarely pubescent. Head relatively large, $0.50-0.65$ times pronotal width, covered with short, greyish pubescence; antenna composed of eight antennomeres, relatively stout, scape asymmetrical, scape and pedicel subequal in length and width, antennomeres 3-7 gradually broadening and elongated, antennomere 8 subconical, distinctly longer than antennomere 7 (Fig. 3e). Mandible unidentate, prostheca indistinct, lateral margin of mandible strongly curved (Fig. 3g). Terminal maxillary palpomere elongate with sides nearly parallel or expanded to apex, rarely subquadrate, apical margin obliquely truncate (Fig. 3f). Terminal labial palpomere subconical (Fig. 3h).

Prothorax. Descending anteriorly. Prosternum T-shaped, in front of coxae distinctly longer than basal width of prosternal process. Prosternal process moderately broad, subparallel or slightly expanded to apex, truncate at apex, without carinae (Fig. 3d). Mesoventrite approximately trapezoidal, anterior margin of mesoventrite straight. Meso-metaventral process narrow, junction straight, with visible suture. Scutellum small and triangular. Elytra distinctly wider than pronotum at base, surface finely or coarsely punctate. Elytral epipleura distinctly oblique, with or without grooves. Abdomen with five ventrites in female and six ventrites in male. Abdominal postcoxal lines incomplete, not curved, posteriorly reaching or very close to hind margin of ventrite. Legs with stout femora, tibiae with a triangular tooth at basal $1 / 3$, without tibial spurs (Fig. 3i-j); tarsal claw with basal tooth (Fig. 3k). 


\section{Key to species of the genus Chilocorus from China}

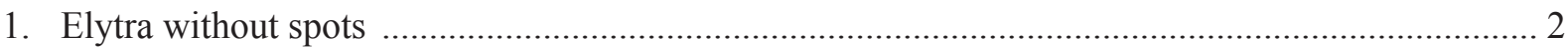

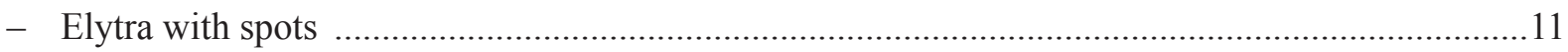

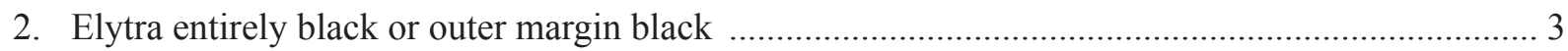

- Elytra entirely red (Fig. 1a) .......................................................................... politus Mulsant, 1850

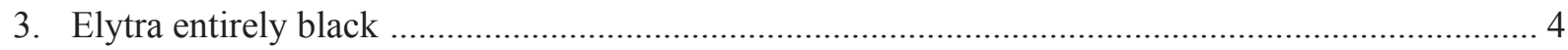

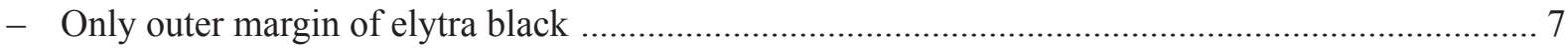

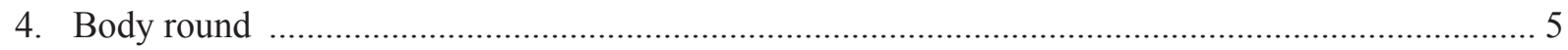

- Body heart-shaped (Fig. 2a) ......................................................... yunlongensis Cao \& Xiao, 1984

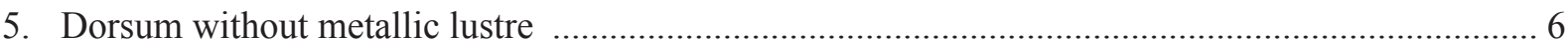

- Dorsum with bluish lustre (Fig. 3a) ........................................... nigricaeruleus Li \& Wang sp. nov.

6. Pronotum mostly black except lateral parts yellow (Fig. 4c) ................. C. nigrita (Fabricius, 1798)

- Pronotum black except anterior angles yellow (Fig. 5c) .................................... me melas Weise, 1898

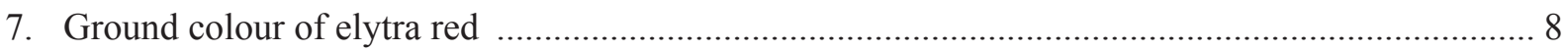

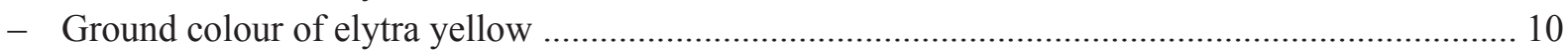

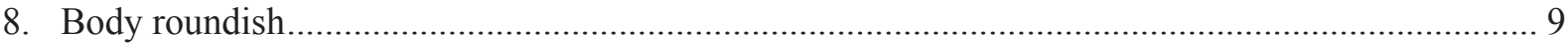

- Body heart-shaped (Fig. 6a .......................................................................... C. rubidus Hope, 1831

9. Scutellum black (Fig. 7a-c); abdominal postcoxal line not reaching posterior margin of abdominal ventrite 1 (Fig. 7d)

C. chinensis Miyatake, 1970

- Scutellum red (Fig. 8a-c); abdominal postcoxal line reaching posterior margin of abdominal ventrite 1 (Fig. 8d) C. rufitarsis Motschulsky, 1853

10. Head black, pronotum and outer margin of elytra black with bluish lustre (Fig. 9a-

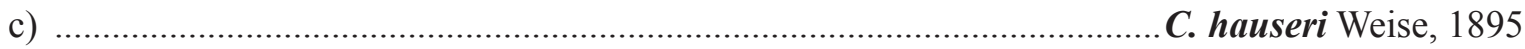

- Head and pronotum yellow, outer margin of elytra black without bluish lustre (Fig. 10a-

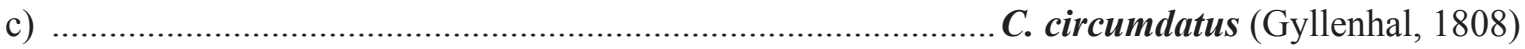

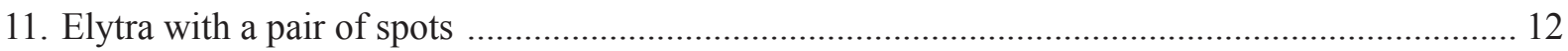

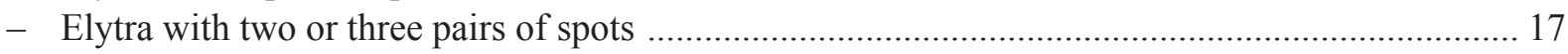

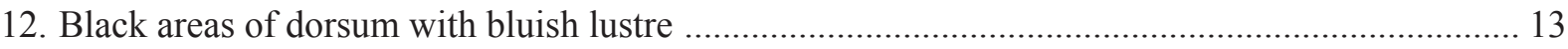

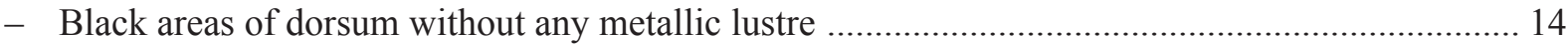

13. Penis guide as long as parameres (Fig. 11h) ....................................... chalybeatus Gorham, 1892

- Penis guide distinctly shorter than parameres (Fig. 12g) ............................... shirozui Sasaji, 1968

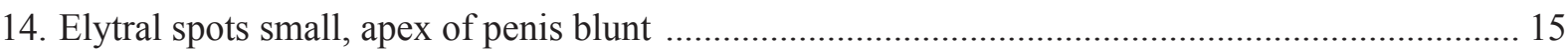

- Elytral spots relatively larger, penis constricted to a pointed tip (Fig. 13a, e-f)

C. hupehanus Miyatake, 1970

15. Penis and tegmen moderately stout 16

- Penis and tegmen strongly stout, penis guide subtriangular, widest at base, gradually converging apically to blunted tip in ventral view (Fig. 14e, h) ................... C. strenotubus Li \& Wang sp. nov.

16. Penis guide approximately $2 / 3$ length of parameres in lateral view, distinctly expanded at basal $1 / 4$ in ventral view (Fig. $15 \mathrm{~g}-\mathrm{h}$ )

C. kuwanae Silvestri, 1909 
- Penis guide approximately $4 / 5$ length of parameres in lateral view, approximately parallel from base to basal $1 / 2$ (Fig. 16g-h) C. esakii Kamiya, 1959

17. Ground colour of elytra black 18

- Ground colour of elytra brown 19

18. Body length: 3.40-3.70 $\mathrm{mm}$, broadly oval, dorsum with bluish lustre (Fig. 17a)

C. alishanus Sasaji, 1968

- Body length: 4.30-5.30 mm, heart-shaped, dorsum without any metallic lustre (Fig. 18a)

C. bijugus Mulsant, 1853

19. Body length: 3.33-3.67 mm, elytra dark brown, with three pairs of roundish yellow spots, arranged in a row before centre, penis guide as long as parameres (Fig. 19a, g)

C. bipustulatus (Linnaeus, 1758)

- Body length: 3.80-4.67 mm, elytra dark brown, with a pair of transverse yellow strips, situated before centre, about $2 / 3$ elytra width, penis guide slightly longer than parameres (Fig. 20a, g)

C. geminus Zaslavskij, 1962

\section{Chilocorus politus Mulsant, 1850}

Figs 1, 21

Chilocorus politus Mulsant, 1850: 455.

Chilocorus politus - Crotch 1874: 184. - Weise 1902: 507. - Miwa 1931: 87. — Korschefsky 1932: 240.— Nagaraja \& Hussainy 1967: 252. — Sasaji 1968a: 20. — Chazeau et al. 1974: 280. — Hoáng 1983: 19.— Booth \& Pope 1989: 360. — Cao et al. 1992: 153. — Poorani 2002: 312. — Kováŕ 2007: 593.

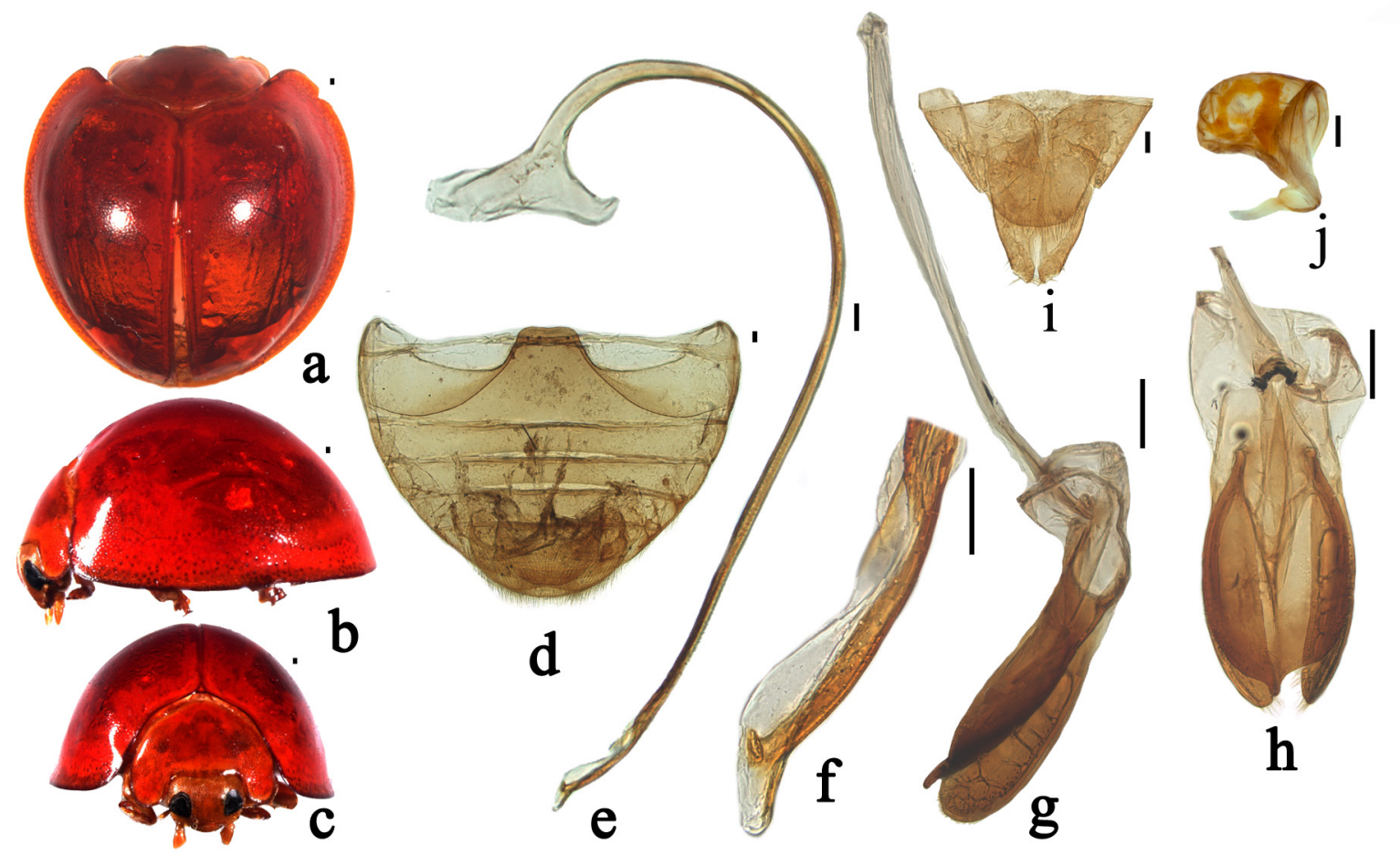

Fig. 1. Chilocorus politus Mulsant, 1850. a. Dorsal view. b. Lateral view. c. Frontal view. d. Abdomen. e. Penis. f. Apex of penis. g. Tegmen, lateral view. h. Tegmen, ventral view. $\mathbf{i}-\mathbf{j}$. Female genitalia. i. Ovipositor. j. Spermatheca. Scale bars: $0.1 \mathrm{~mm}$. (SCAU) 


\section{Material examined}

CHINA: Guangxi Zhuang Autonomous Region: 1 ô, Hongqilinchang, Shiwandashan, 9 Nov. 2004, X.M. Wang leg. (SCAU 20051206200).

NEPAL: 1 ô, 1 q, Tansen, Palpa, Lumbini, 16 Oct. 2011, S.X. Ren leg. (SCAU).

\section{Distribution}

China (Guangxi, Yunnan, Tibet, Taiwan) (Fig. 21), Thailand, Laos, India, Nepal, Bhutan, Indonesia.

\section{Chilocorus yunlongensis Cao \& Xiao, 1984}

Figs 2, 21

Chilocorus yunlongensis Cao \& Xiao, 1984: 117

Chilocorus yunlongensis - Cao et al. 1992: 159. — Pang et al. 2004: 29. — Kováŕ 2007: 594.

\section{Type material}

\section{Holotype}

CHINA: Ô, Yunnan Prov., Yunlong, 2800 m a.s.1., 24 May 1980, collector unknown (FIQB 83010).

\section{Paratypes}

CHINA: 3 우, same collection data as for holotype (FIQB).

\section{Distribution}

China (Yunnan) (Fig. 21).

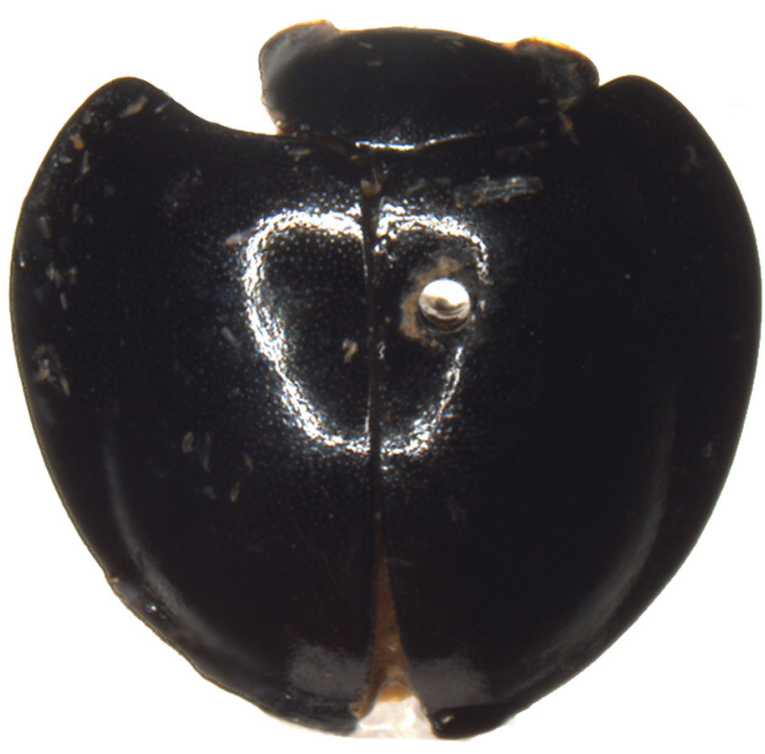

a
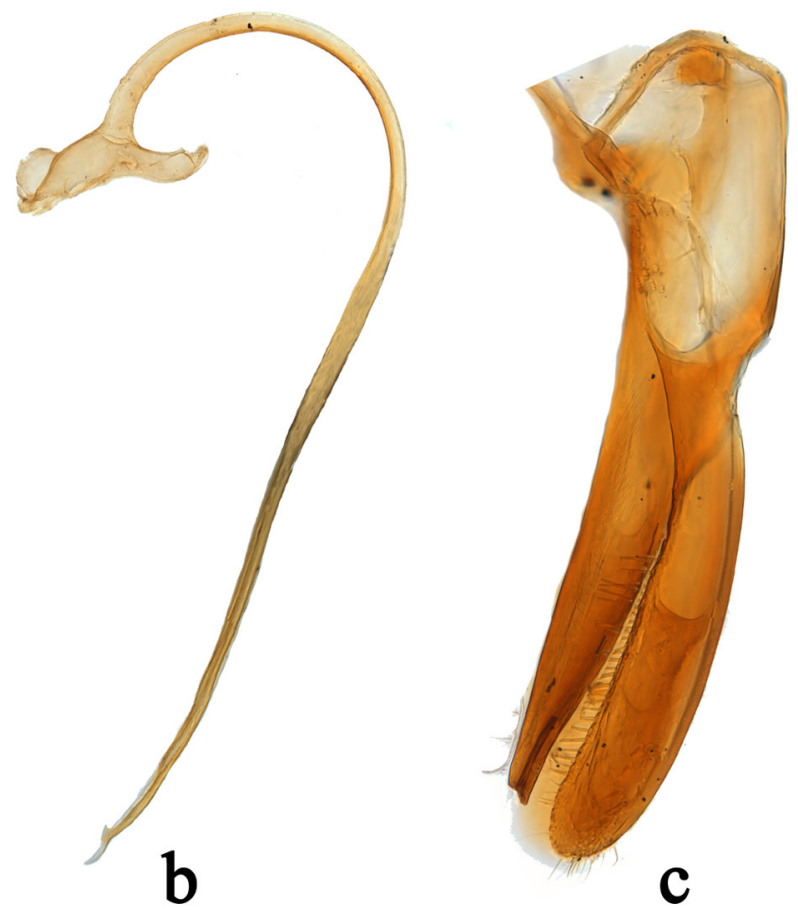

Fig. 2. Chilocorus yunlongensis Cao \& Xiao, 1984. a. Dorsal view. b. Penis. c. Tegmen, lateral view. Scale bars: $0.1 \mathrm{~mm}$. (FIQB) 
Chilocorus nigricaeruleus Li \& Wang sp. nov. urn:lsid:zoobank.org:act:AD963BB9-B700-4984-BE32-C9AFAFFE15D1

Figs 3, 21

\section{Diagnosis}

This species resembles Chilocorus melas Weise, 1898, but can be distinguished from it by having relatively large black body with dorsum with bluish lustre (Fig. 3a) and equal length of penis guide and parameres (Fig. 3o). In C. melas, the body is relatively small, dorsum black (Fig. 5a) and the penis guide distinctly longer than parameres (Fig. $5 \mathrm{~g}$ ).

\section{Etymology}

The specific epithet is derived from the Latin and refers to the colour of the elytra and pronotum, black with a bluish lustre.

\section{Type material}

\section{Holotype}

CHINA: Ō, Yunnan Prov., Longtan, Ximeng, 900 m a.s.1., 9-10 May 2008, X.M. Wang leg. (SCAU (E) 11287).

\section{Paratypes}

CHINA: 1 ôn, same collection data as for holotype (SCAU (E) 11288).

\section{Description (male)}

Measurements. TL: $4.40-4.67 \mathrm{~mm}$, TW: $4.00-4.33 \mathrm{~mm}$, TH: $2.33-2.40 \mathrm{~mm}$, TL/TW: $1.08-1.10$, PL/ PW: $0.48-0.51$, EL/EW: 0.98-0.99.

BoDy. Roundish, strongly convex. Head, antenna and mouthparts yellow, sparsely covered with short, greyish pubescence. Pronotum black with bluish lustre, except anterior angles yellow. Scutellum and elytra black with bluish lustre (Fig. 3a-c). Underside yellow except elytral epipleura black, metaventrite brownish yellow, sparsely covered with short, greyish pubescence. Head relatively large, $0.52 \times$ as wide as pronotum, punctures on frons large and densely distributed, 1.0-2.0 diameters apart, surface polished between punctures. Eyes approximately oval, densely faceted, interocular distance $0.41 \times$ as wide as head (Fig. 3c). Pronotum $0.51 \times$ as wide as elytra, pronotal punctures moderately large and moderately densely distributed, smaller than those on head, 2.0-3.0 diameters apart, surface polished between punctures. Punctures on elytra moderately large and sparsely distributed, 2.0-4.0 diameters apart, similar to those on pronotum. Prosternal process moderate broad, slightly expanded to apex. Abdominal postcoxal lines incomplete, reaching posterior margin of abdominal ventrite 1 and running along posterior margin, then almost reaching lateral margin. Posterior margin of male abdominal ventrite 5 broadly rounded and ventrite 6 emarginate medially (Fig. 31).

Male genitalia. Penis slender, penis capsule with long outer and inner arms, apex of penis slightly narrow with membranous appendage (Fig. $3 m-n$ ). Tegmen stout, penis guide widest at basal $1 / 3$, then gradually converging to blunt apex, distinctly asymmetrical in ventral view and widest at base, gradually converging to blunt apex, bent outwardly from apical $1 / 6$ to apex in lateral view. Parameres constricted from base to basal $1 / 2$ than expanded toward apex, as long as penis guide, densely covered with short setae at the inner surfaces and distal end in lateral view (Fig. 3o-p).

\section{Distribution}

China (Yunnan) (Fig. 21). 


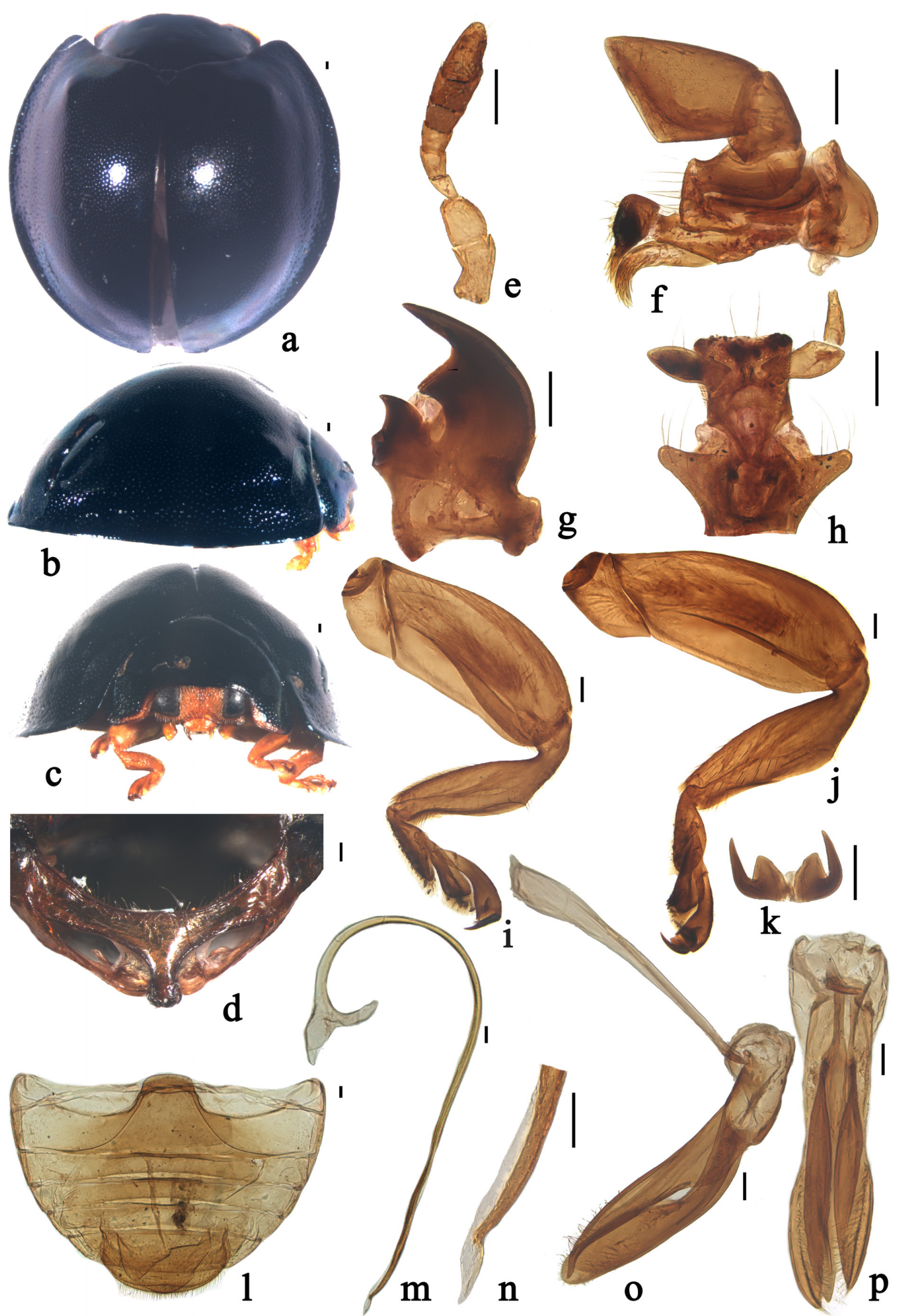

Fig. 3. Chilocorus nigricaeruleus Li \& Wang sp. nov., holotype. a. Dorsal view. b. Lateral view. c. Frontal view. d. Prothorax. e. Antenna. f. Maxilla. g. Mandible. h. Labium. i. Front leg. j. Hind leg. k. Tarsal claw. l. Abdomen. m. Penis. n. Apex of penis. o. Tegmen, lateral view. p. Tegmen, ventral view. Scale bars: $0.1 \mathrm{~mm}$. (SCAU (E) 11287) 
Chilocorus nigrita (Fabricius, 1798)

Figs 4, 21

Coccinella nigrita Fabricius, 1798: 79.

Chilocorus nigrita - Bielawski 1957: 86. - Booth 1998: 362. — Poorani 2002: 312.

Chilocorus nigritus - Mulsant 1850: 463. — Crotch 1874: 184. — Korschefsky 1932: 240. — Nagaraja \& Hussainy 1967: 252. — Leeper 1976: 287. — Kováŕ 2007: 593.

non Chilocorus nigritus - Liu 1963: 78.

\section{Material examined}

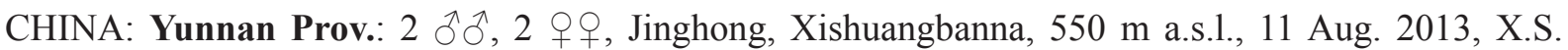
Chen leg. (SCAU); 2 q 9 , Ruili, 20 Oct. 2000, Z.Q. Peng leg. (SCAU); 2 q , Dengshandao, Ruili, 22 Oct. 2000, Z.Q. Peng leg. (SCAU).

\section{Distribution}

China (Yunnan) (Fig. 21), Far East, Indian subcontinent, Oriental region, Australian region, USA, Brazil, Africa.

Chilocorus melas Weise, 1898

Figs 5, 21

Chilocorus melas Weise, 1898b: 229.

Chilocorus gressitti Miyatake, 1970: 330.

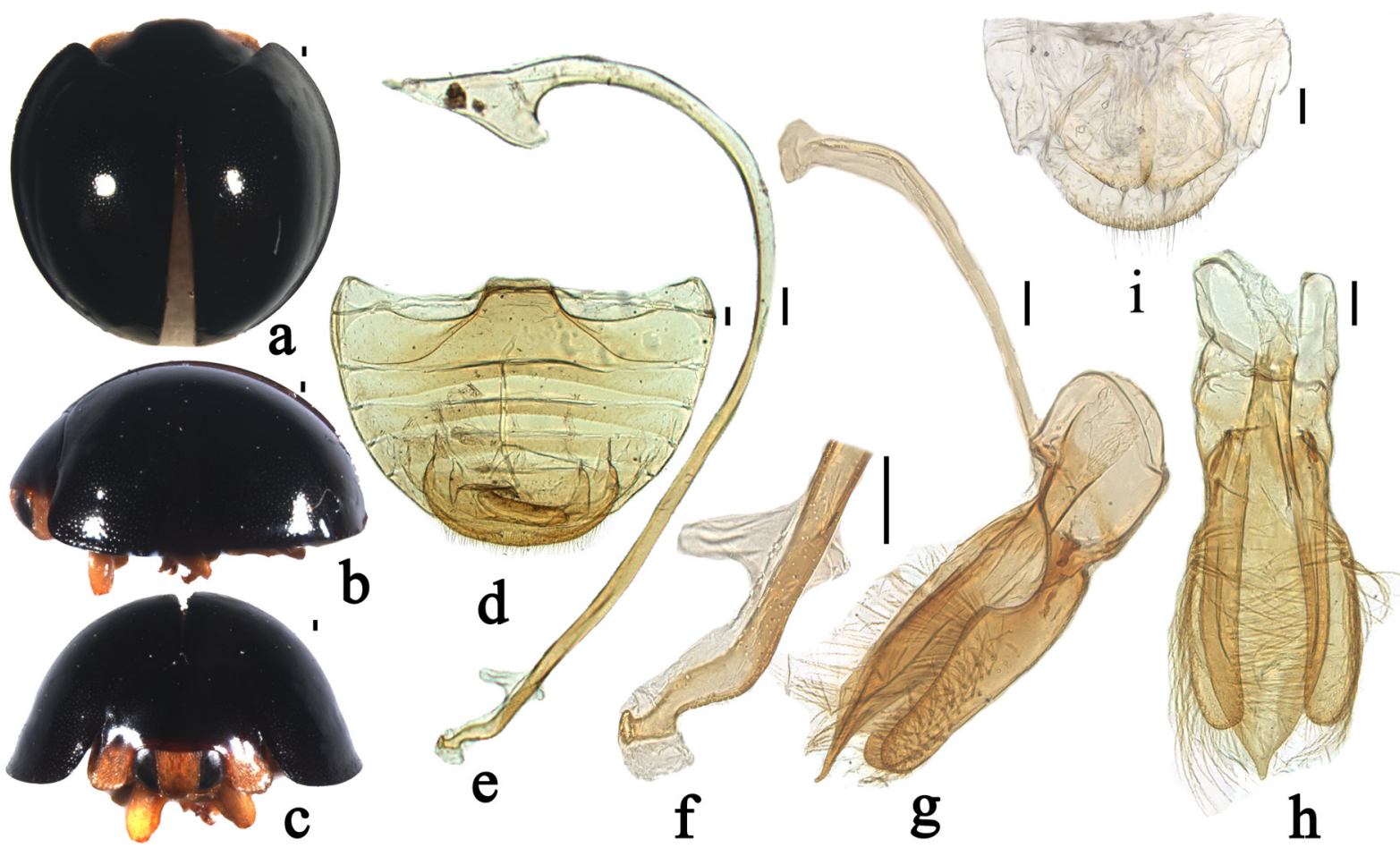

Fig. 4. Chilocorus nigrita (Fabricius, 1798). a. Dorsal view. b. Lateral view. c. Frontal view. d. Abdomen. e. Penis. f. Apex of penis. g. Tegmen, lateral view. h. Tegmen, ventral view. i. ovipositor. Scale bars: $0.1 \mathrm{~mm}$. (SCAU) 
Chilocorus melas - Korschefsky 1932: 243. — Booth 1998: 364. — Poorani 2002: 311. — Pang et al. 2004: 28. — Kováŕ 2007: 593. - Ren et al. 2009: 132.

Chilocorus gressitti - Pang \& Mao 1979: 84. — Hoáng 1983: 18. — Jing 1992: 567. — Cao et al. 1992: 159. - Pang et al. 2002: 317.

Chilocorus nigritus - Liu 1963: 78.

\section{Material examined}

CHINA: Guangdong Prov.: 1 , Tianma, Xinhui, 24 Apr. 1956, Z.B. Zhou leg. (SCAU); 4 $q$, Xinhui, Nov. 1956, collector unknown (SCAU); 1 q, Nanhai, 14 Oct. 1955, L.B. Huo leg. (SCAU). - Guangxi Prov.: 1 Ô, Longsheng, 6 Apr. 1974, Y.L. Luo leg. (SCAU); 1 ô, Nanning, 4 Aug. 1985, X.F. Pang leg. (SCAU). - Fujian Prov.: 1 đ̃, Huaan, 26 Apr. 1982, Y.Q. Tang leg. (SCAU). - Hainan Prov.: 1 đ; 3 우, Diaoluoshan, Jul. 1995, Z.Q. Peng leg. (SCAU); 1 O, Diaoluoshan, 8 May 2005, X.M. Wang leg. (SCAU); 1 ô, Dongfang, 27 Nov. 1997, Z.Q. Peng leg. (SCAU).

\section{Distribution}

China (Fujian, Guangdong, Guangxi, Hainan, Sichuan, Yunnan, Hongkong) (Fig. 21), Burma, Thailand, Laos, India, Nepal, Bhutan, Indonesia.

\section{Chilocorus rubidus Hope, 1831}

Figs 6, 21

Chilocorus rubidus Hope, 1831: 31.

Coccinella tristis Faldermann, 1835: 452.

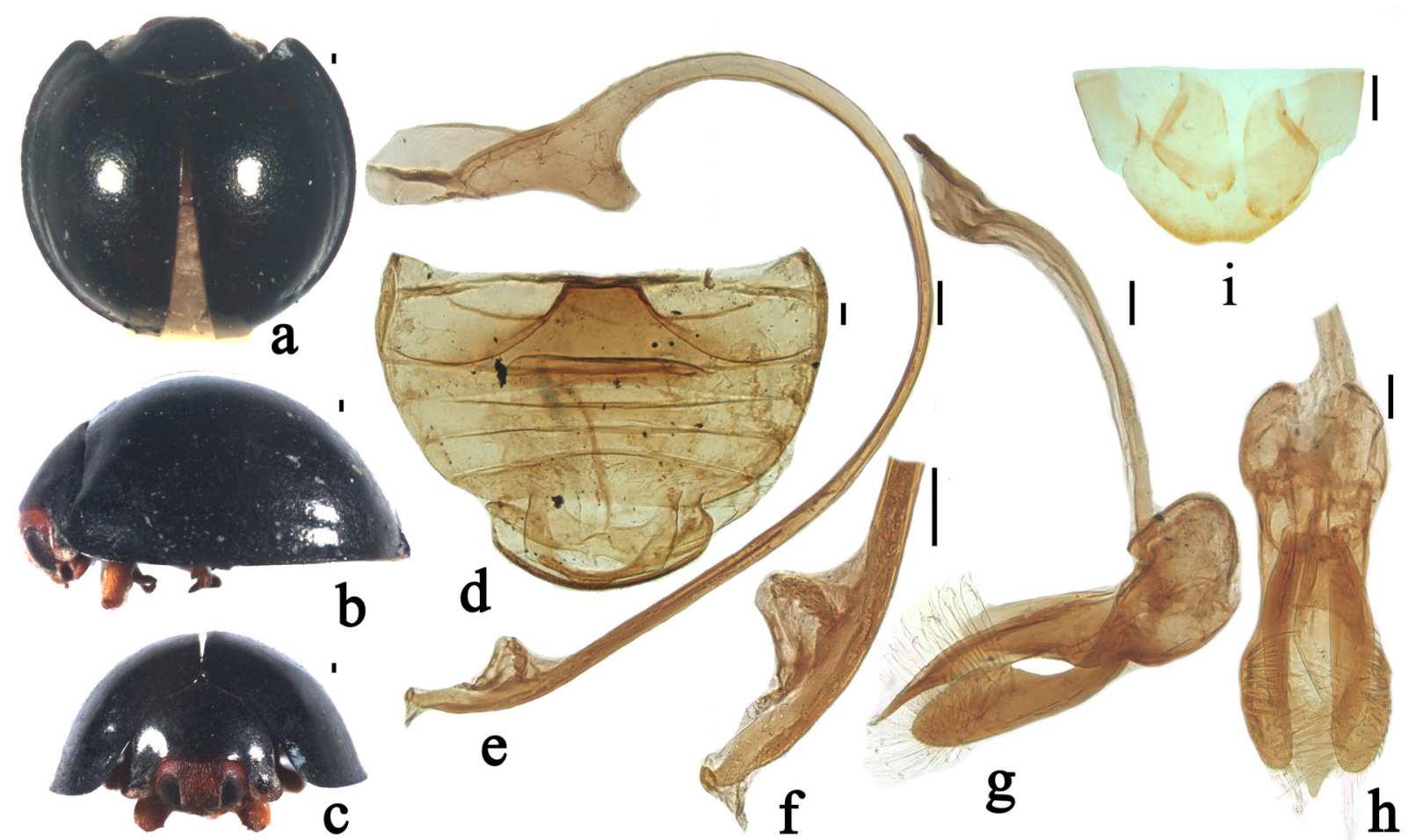

Fig. 5. Chilocorus melas Weise, 1898. a. Dorsal view. b. Lateral view. c. Frontal view. d. Abdomen. e. Penis. f. Apex of penis. g. Tegmen, lateral view. h. Tegmen, ventral view. i. ovipositor. Scale bars: $0.1 \mathrm{~mm}$. (SCAU) 
Chilocorus rubidus - Mulsant 1850: 452. - Crotch 1874: 183. - Weise 1887: 210. — Blackburn 1889: 1275. — Lewis 1896: 31. — Korschefsky 1932: 241. - Hu 1937: 563. — Mader 1955b: 775. — Kapur 1956: 262; 1972: 313. — Kamiya 1959: 100. — Nakane 1963: 209. — Liu 1963: 75. — Nagaraja \& Hussainy 1967: 253. — Miyatake 1970: 318. — Sasaji 1971: 224. — Pang \& Mao 1979: 80. — Booth \& Pope 1989: 362. — Jing 1992:567. — Cao et al. 1992:154. — Pang et al. 2002: 320; 2004: 29. — Poorani 2002: 311. — Kovář 2007: 593. — Ren et al. 2009: 134.

Chilocorus tristis - Mulsant 1850: 452. — Crotch 1874: 183. — Weise 1885: 51. — Lewis 1873: 56.

Chilocorus rubidus ab. tristis - Korschefsky 1932: 241. — Kapur 1956: 262.

\section{Material examined}

CHINA: Inner Mongolia: 1 ภ, 1 †, Xiangchizi, Gulaben, 1861-2283 m a.s.1., 7 Aug. 2010, C.W. Li leg. (SCAU). - Ningxia Hui Autonomous Region: $3 \hat{\partial} \widehat{\partial}, 1$ , Suyukou, Helanshan, Yinchuan 1950 m a.s.1., 12 Aug. 2009, X.M. Wang leg. (SCAU). - Jiangsu Prov.: 1 , Nanjing University, 5 Sep. 1962, collector unknown (SCAU); 2 ô, Nanjing Agricultural College, time of collection and collector unknown (SCAU). - Zhejiang Prov.: 1 ô, Hangzhou, time of collection unknown, X. Cong leg. (SCAU). - Shandong Prov.: 1 \&, Yuantoucun, Qingdao, 7 Jun. 1958, collector unknown (SCAU). -

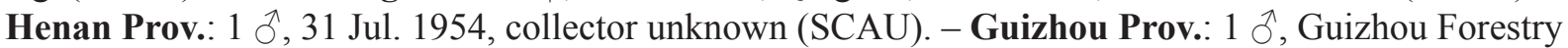
Institute, time of collection and collector unknown (SCAU). - Yunnan Prov.: 1 ô, 4 q $q$, Yuxi, Jul. 1981, collector unknown (SCAU); 1 §ે, Jilong, Tibet, 25 Jul. 1984, Z.X. Yan leg. (SCAU).

\section{Distribution}

China (Beijing, Tianjin, Hebei, Inner Mongolia, Liaoning, Jilin, Heinongjiang, Jiangsu, Zhejiang, Fujian, Shandong, Henan, Hunan, Hainan, Sichuan, Guizhou, Yunnan, Tibet, Shannxi, Gansu, Ningxia) (Fig. 21), Mongolia, Korea, Japan, India, Nepal, Indonesia, Siberia.

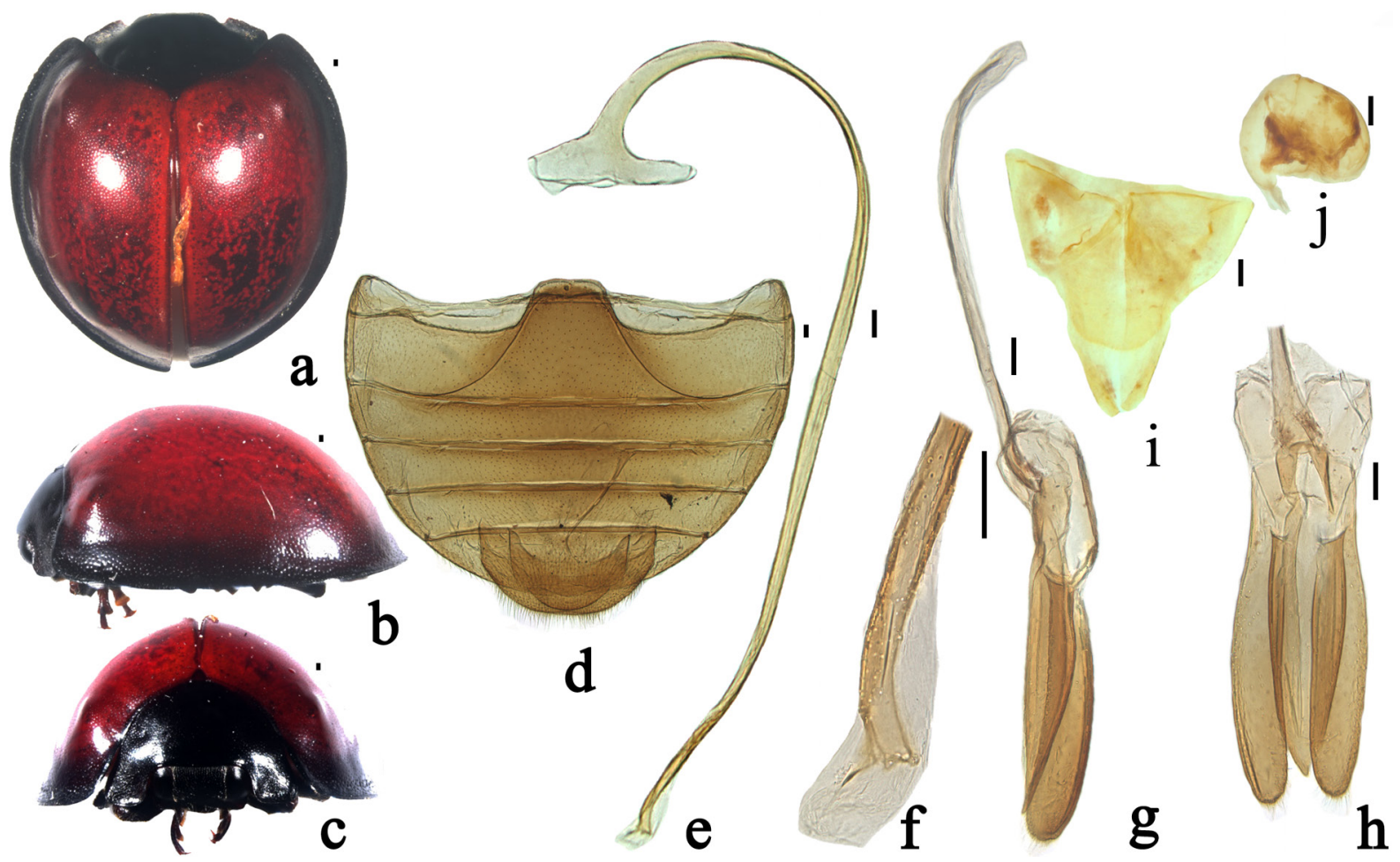

Fig. 6. Chilocorus rubidus Hope, 1831. a. Dorsal view. b. Lateral view. c. Frontal view. d. Abdomen. e. Penis. f. Apex of penis. g. Tegmen, lateral view. h. Tegmen, ventral view. i-j. Female genitalia: i. ovipositor. j. spermatheca. Scale bars: $0.1 \mathrm{~mm}$. (SCAU) 
Chilocorus chinensis Miyatake, 1970

Figs 7, 21

Chilocorus chinensis Miyatake, 1970: 322.

Chilocorus chinensis - Pang \& Mao 1979: 81. — Hoáng 1983: 22. — Cao et al. 1992: 156. — Pang et al. 2002: 317; 2004: 28. — Kovář 2007: 593. — Ren et al. 2009: 128.

\section{Material examined}

CHINA: Henan Prov.: 1 q, Longyuwan, Luoyang, 1390 m a.s.1., 11-12 Nov. 2009, X.M. Wang leg. (SCAU). - Guangdong Prov.: 2 đิ $\widehat{\jmath}$, Huangniping, Shimentai, 7 Oct. 2004, X.M. Wang leg. (SCAU). Anhui Prov.: 1 T, Tangkouzhen, Huangshan, 12-13 Sep. 2010, X.M. Wang leg. (SCAU). - Guizhou Prov.: 1 ô, Xianheping, Anlong, 14-15 Sep. 2006, X.M. Wang leg. (SCAU). - Hainan Prov.: 1 q, Bawangling, 21 Mar. 1996, Z.Q. Peng leg. (SCAU). - Jiangsu Prov.: 2 specimens, Agricultural college, Nanjing, the time of collection and collector unknown (SCAU). - Jiangxi Prov.: 2 ô ô, 1 , , Maoping Jinggangshan, 20 Sep. 2004, X.M. Wang leg. (SCAU); 4 specimens, Lushan, Sep. 1978, Y.H. Wang leg. (SCAU).

\section{Distribution}

China (Anhui, Zhejiang, Fujian, Jiangxi, Henan, Guangdong, Guangxi, Hainan, Guizhou, Yunan, Jiangsu) (Fig. 21).

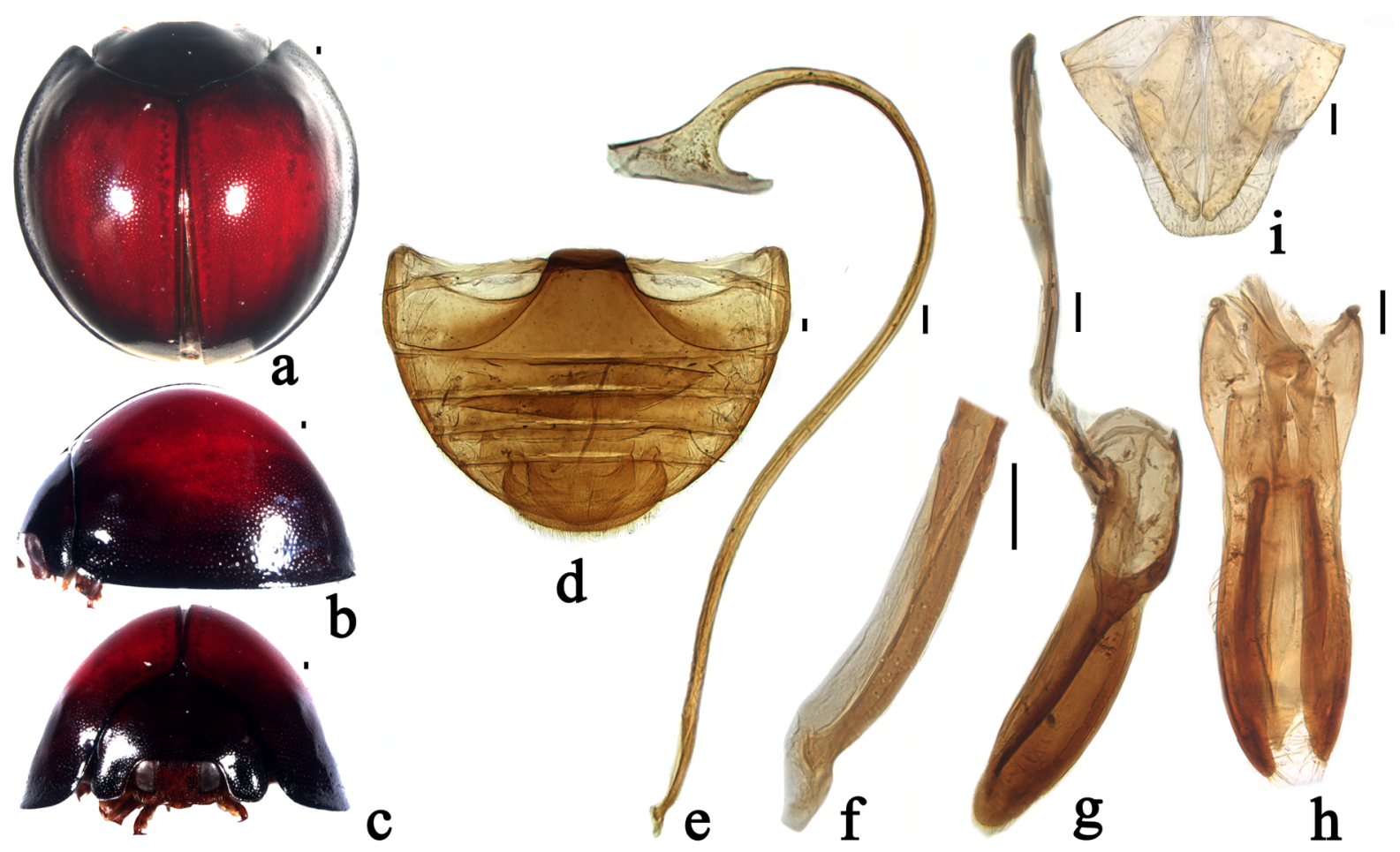

Fig. 7. Chilocorus chinensis Miyatake, 1970. a. Dorsal view. b. Lateral view. c. Frontal view. d. Abdomen. e. Penis. f. Apex of penis. g. Tegmen, lateral view. h. Tegmen, ventral view. i. Ovipositor. Scale bars: $0.1 \mathrm{~mm}$. (SCAU) 
Chilocorus rufitarsis Motschulsky, 1853

Figs 8, 21

Chilocorus rufitarsis Motschulsky, 1853: 50.

Chilocorus rufitarsis - Crotch 1874: 183. - Korschefsky 1932: 243. - Hu 1937: 563. - Mader 1955b: 775. — Liu 1963: 79. — Miyatake 1970: 319. — Pang \& Mao 1979: 80. — Hoáng 1983: 22. — Cao et al. 1992: 155. — Pang et al. 2002: 320; 2004: 29. — Kováŕ 2007: 594. — Ren et al. 2009: 134.

\section{Material examined}

CHINA: Hunan Prov.: 1 o, Shennonggu, Yanlin, 650-800 m a.s.1., 9 Oct. 2010, X.M. Wang leg. (SCAU). - Shandong Prov.: 1 q, Qishui, Jul. 1958, collector unknown (SCAU). - Guizhou Prov.: 2 đ ${ }^{\lambda}$, 1 , , Guizhou Forestry Institute, time of collection and collector unknown (SCAU). - Guangdong Prov.: 1 Ĵ, Dadongshan, Liangzhou, 19 Jul. 2005, X.M. Wang leg. (SCAU); 1 specimen, Shipai, 7 Apr. 1954, J.X. Jiang leg. (SCAU); 1 specimen, Shipai, 16 Oct. 1960, Y.K. Wang leg. (SCAU); 1 specimen, Nanhai, Mar. 1960, collector unknown (SCAU); 2 specimens, Zhangdongshui, 26 Sep. 1989, J.C. Wu leg.

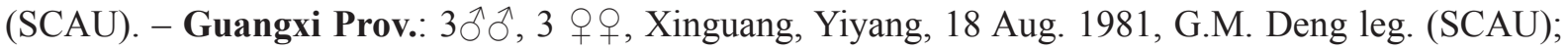
1 q, Jun. 1960, T.S. Pu leg.; 2 우, Quanzhou, 1980, collector unknown (SCAU).

\section{Distribution}

China (Jiangsu, Zhejiang, Fujian, Jiangxi, Hunan, Guangdong, Guangxi, Sichuan, Guizhou, Yunnan, Hongkong, Shandong) (Fig. 21), Vietnam.

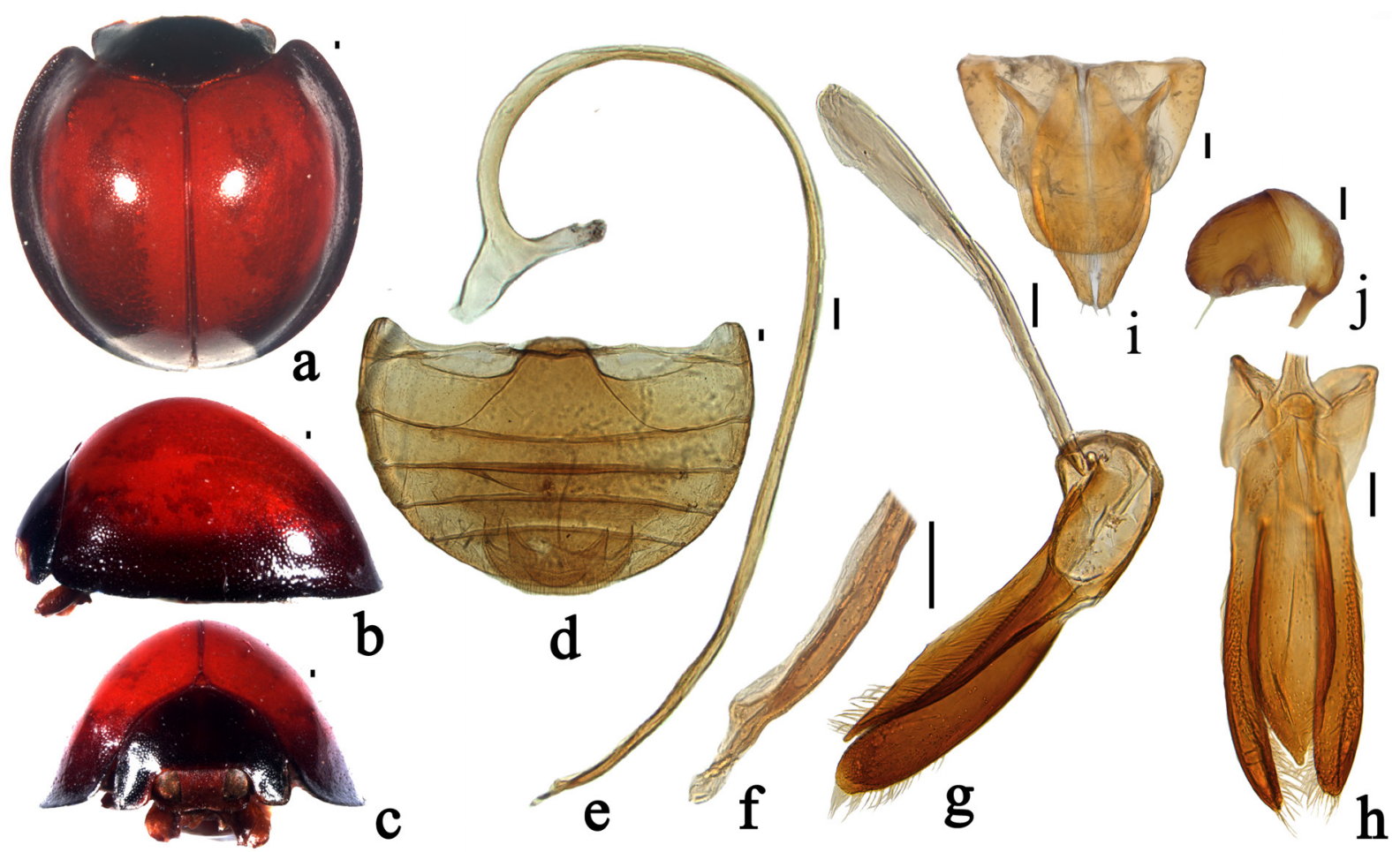

Fig. 8. Chilocorus rufitarsis Motschulsky, 1853. a. Dorsal view. b. Lateral view. c. Frontal view. d. Abdomen. e. Penis. f. Apex of penis. g. Tegmen, lateral view. h. Tegmen, ventral view. i-j. Female genitalia. i. Ovipositor. j. Spermatheca. Scale bars: $0.1 \mathrm{~mm}$. (SCAU) 
Chilocorus hauseri Weise, 1895

Figs 9, 21

Chilocorus hauseri Weise, 1895a: 135.

Chilocorus hauseri - Sicard 1913: 500. — Korschefsky 1932: 243. — Liu 1963: 77. — Nagaraja \& Hussainy 1967: 250. — Pang \& Mao 1979: 82. — Cao et al. 1992: 156. — Pang et al. 2002: 318; 2004: 28. — Kováŕ 2007: 593. — Ren et al. 2009: 130.

\section{Material examined}

CHINA: Hainan Prov.: 1 d , 2 우, Jianfengling, 15 Jun. 1982, Y.F. Liu leg. (SCAU). - Sichuan Prov.: $3 \widehat{\jmath}$, Puge, $2000 \mathrm{~m}$ a.s.1., 15 Sep. 2007, X.M. Wang leg. (SCAU). - Yunnan Prov.: 1 ô, 1 q, Funing, 16 Aug. 2005, X.M. Wang leg. (SCAU); 1 Ô, Tongbiguan, Nabang, Yingjiang, 1000 m a.s.1., 22-23 May 2008, X.M. Wang leg. (SCAU).

\section{Distribution}

China (Fujian, Guangdong, Hainan, Sichuan, Yunnan) (Fig. 21), Burma, India (Sikkim).

\section{Chilocorus circumdatus (Gyllenhal, 1808)}

Figs 10, 21

Coccinella circumdatus Gyllenhal in Schönherr, 1808: 152.

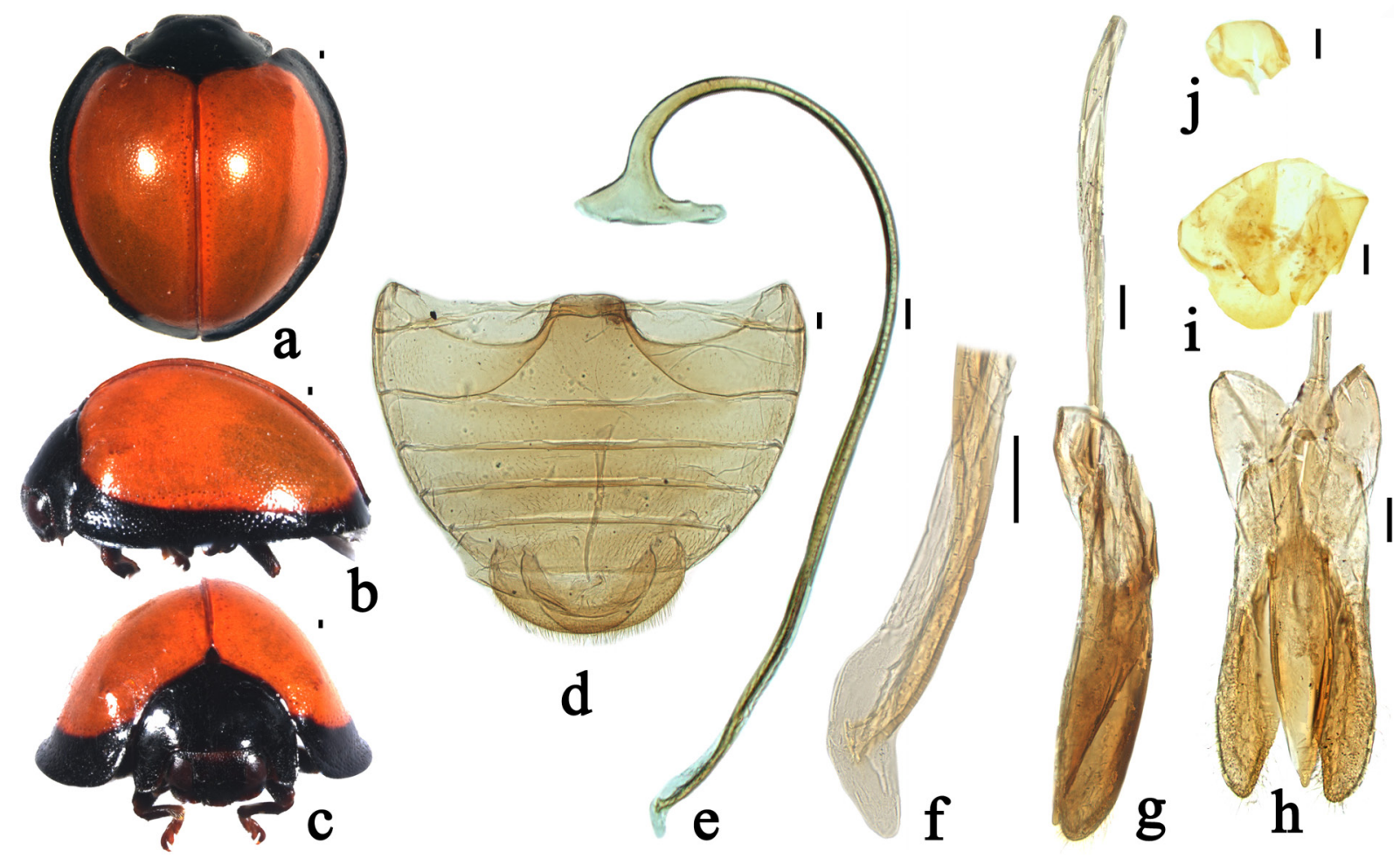

Fig. 9. Chilocorus hauseri Weise, 1895. a. Dorsal view. b. Lateral view. c. Frontal view. d. Abdomen. e. Penis. f. Apex of penis. g. Tegmen, lateral view. h. Tegmen, ventral view. $\mathbf{i}-\mathbf{j}$. Female genitalia. i. Ovipositor. j. Spermatheca. Scale bars: $0.1 \mathrm{~mm}$. (SCAU) 
Chilocorus circumdatus - Mulsant 1850: 454. - Crotch 1874: 186. - Korschefsky 1932: 242. Mader 1955b: 867. — Liu 1963: 77. — Nagaraja \& Hussainy 1967: 250. — Miyatake 1970: 323. — Leeper 1976: 287. — Pang \& Mao 1979: 83. — Hoáng 1983: 19. — Cao et al. 1992:158. — Pang et al. 2002: 317; 2004: 28. — Poorani 2002: 311.—Kováŕ 2007: 593. — Ren et al. 2009: 130.

\section{Material examined}

CHINA: Guangdong Prov.: 1 specimen, Huizhou, 11 May 1988, X.L. Tong leg. (SCAU); 1 specimen, Shenshan, Huidong, 5 May 1988, X.L. Tong leg. (SCAU); 3 specimens, Kuangbianyuan, Yangcun, Jun. 1979 (SCAU); 4 specimens, Shipai, Mar. 1961 (SCAU); 1 specimen, Shipai, Aug. 1956, N.C. Sun leg. (SCAU); 5 specimens, Shipai, Oct. 1956 (SCAU); 1 specimen, Zengcheng, 28 May 1951 (SCAU); $1 \hat{\jmath}$, Huangniping, Shimentai, Guangzhou, 7 Oct. 2004, X.M. Wang leg. (SCAU). - Hainan Prov.: 8 $\widehat{\partial} \widehat{\jmath}$, 3 우, Diaoluoshan, Jul. 1995, Z.Q. Peng leg. (SCAU); 16 specimens, Diaoluoshan, Jul. 1995, Z.Q. Peng leg. (SCAU); 1 Ĵ, Diaoluoshan, 9 May 1995, Z.Q. Peng leg. (SCAU); 24 specimens, Nanfengzhen, Danzhou,Aug. 1995, Z.Q. Peng leg. (SCAU); 4 specimens, Liangyuan, Danzhou, Aug. 1995, Z.Q. Peng leg. (SCAU); 1 đ̂, Yanglan, Sanya, 22 Sep. 1997, Z.Q. Peng leg. (SCAU); 1 Oૈ, Wushi, 14 Jul. 1999 , Z.Q. Peng leg. (SCAU). - Yunnan Prov.: 1 ô, Mengmao, 21 Oct. 2000, Z.Q. Peng leg. (SCAU).

\section{Distribution}

China (Zhejiang, Fujian, Guangdong, Guangxi, Hainan, Yunnan, Hong Kong) (Fig. 21), Indonesia, India, Sri Lanka. Introduced to Australia, America.

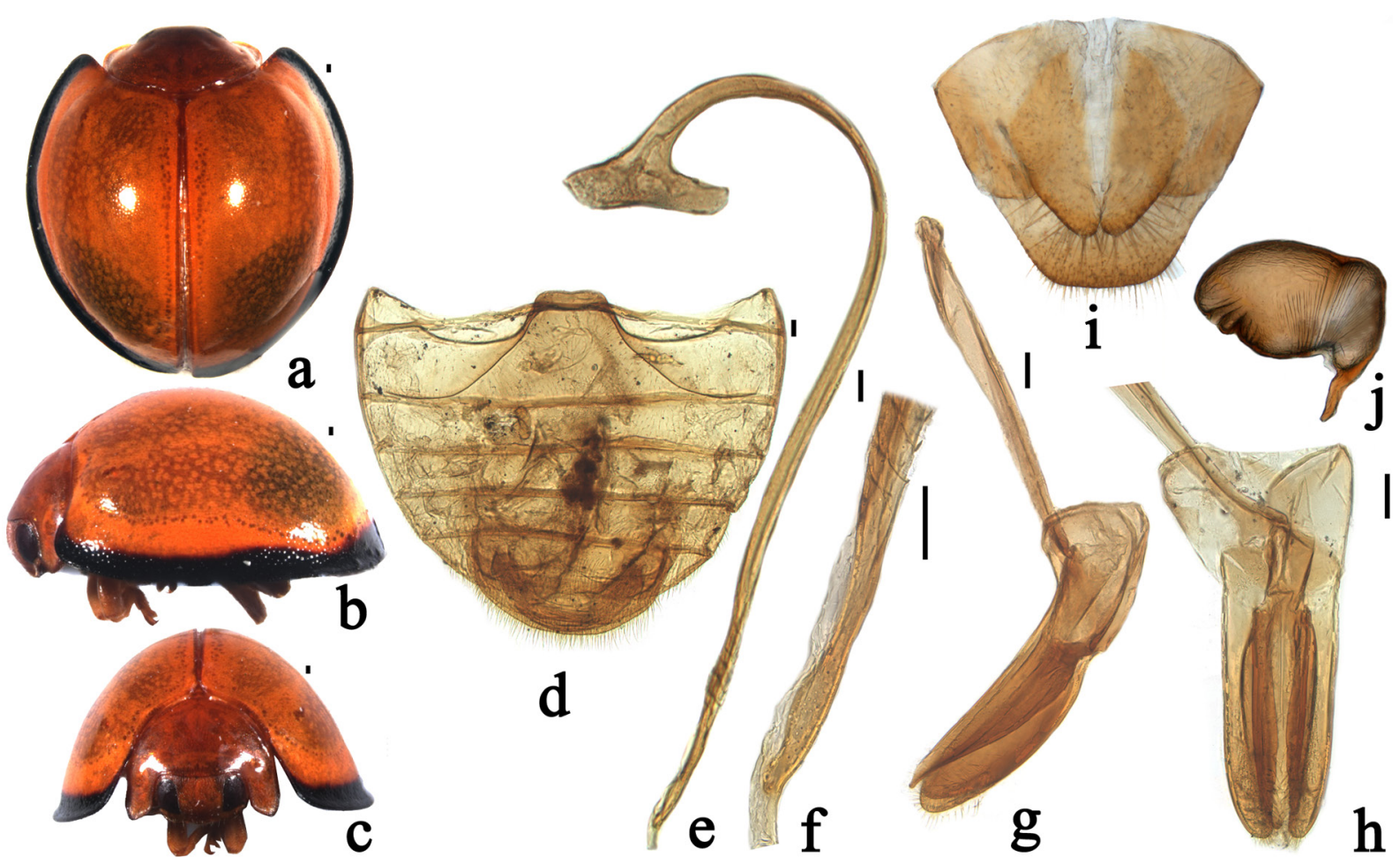

Fig. 10. Chilocorus circumdatus (Gyllenhal, 1808). a. Dorsal view. b. Lateral view. c. Frontal view. d. Abdomen. e. Penis. f. Apex of penis. g. Tegmen, lateral view. h. Tegmen, ventral view. i-j. Female genitalia. i. Ovipositor. j. Spermatheca. Scale bars: $0.1 \mathrm{~mm}$. (SCAU) 
Chilocorus chalybeatus Gorham, 1892

Figs 11, 22

Chilocorus chalybeatus Gorham, 1892: 24.

Chilocorus chalybeatus - Miyatake 1970: 325. — Pang \& Mao 1979: 84. — Hoáng 1983: 21. — Yu et al. 1993: 489. — Pang et al. 2002: 316; 2004: 27. — Kováŕ 2007: 593. — Ren et al. 2009: 128.

\section{Material examined}

CHINA: Zhejiang Prov.: 1 ภ, Tianmu Mountain, Linan, 450-1000 m a.s.1., 18 Sep. 2010, X.M. Wang

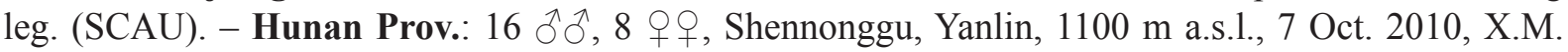
Wang leg. (SCAU). - Guangxi Prov.: 1 đ̃, Shilidaxiagu, Maoer Mountain, 19 Oct. 2004, X.M. Wang leg. (SCAU). - Guizhou Prov.: 1 q, Dongtang, Maolan, Libo, 730 m a.s.1., 15-18 Oct. 2008, J.B Liang leg. (SCAU). - Anhui Prov.: 1 O , Tianju Mountain, 29 Jul. 2005, X.M. Wang leg. (SCAU); 1 , Tianma Jinzhai, $600 \mathrm{~m}$ a.s.1., 3-5 Oct. 2010, X.M. Wang leg. (SCAU). - Gansu Prov.: 1 ð, Dangchuan, Tianshuimaiji, 1800 m a.s.l., 4 Aug. 2009, X.M. Wang leg. (SCAU); 1 ô, Maiji Mountain, Tianshui, 1500 m a.s.1., 17 Oct. 2007, S.X. Ren leg. (SCAU). - Shannxi Prov.: 2 $\widehat{\partial}$, Fengyulinchang, Changan, $1600 \mathrm{~m}$ a.s.1., 27 Jul. 2007, X.M. Wang leg. (SCAU); $2 \hat{\jmath}, 1$, , Longtan village, Fenghuangguzhen,

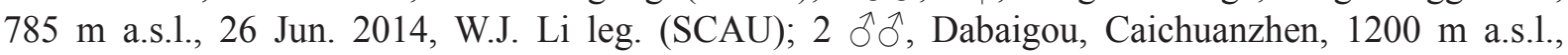
1 Jul. 2014, W.J. Li leg. (SCAU).

\section{Distribution}

China (Anhui, Zhejiang, Fujian, Hunan, Guangdong, Hainan, Sichuan, Guizhou, Yunnan, Shannxi, Gansu, Guangxi) (Fig. 22).

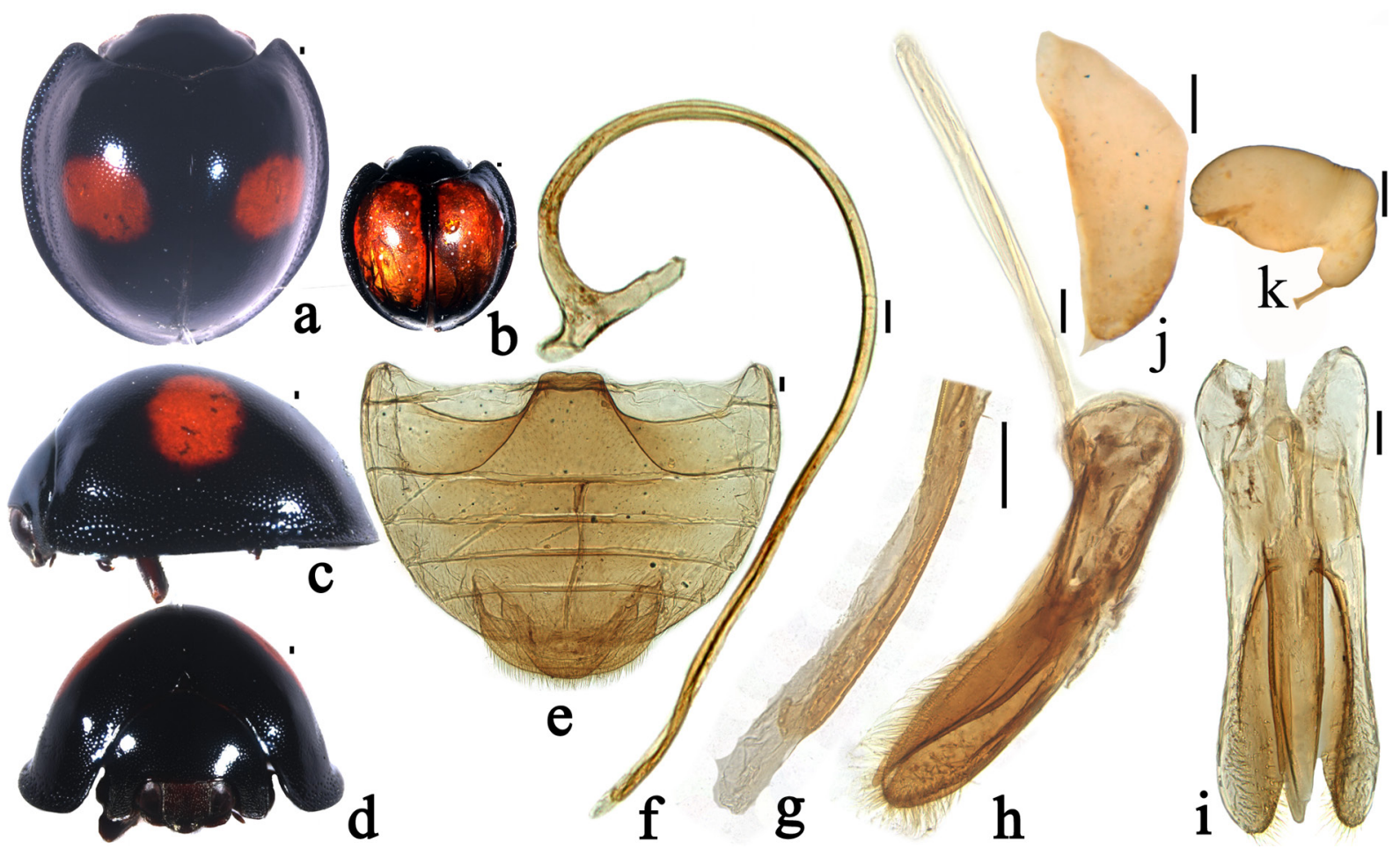

Fig. 11. Chilocorus chalybeatus Gorham, 1892. a-b. Dorsal view. c. Lateral view. d. Frontal view. e. Abdomen. f. Penis. g. Apex of penis. h. Tegmen, lateral view. i. Tegmen, ventral view. j-k. Female genitalia. j. Coxit. k. Spermatheca. Scale bars: $0.1 \mathrm{~mm}$. (SCAU) 
Chilocorus shirozui Sasaji, 1968a: 22.

Chilocorus shirozui - Miytake 1970: 327. — Pang et al. 2004: 29. — Kovář 2007: 594. — Yu 2011: 69.

\section{Material examined}

CHINA: Taiwan Prov.: 2 ふぇ, 4 $q$, Puli (NCHU).

\section{Distribution}

China (Taiwan) (Fig. 22).

Chilocorus hupehanus Miyatake, 1970

Figs 13, 22

Chilocorus hupehanus Miyatake, 1970: 329.

Chilocorus hupehanus - Pang \& Mao 1979: 86. — Pang et al. 2002: 318; 2004: 28. — Kováŕ 2007: 593. - Ren et al. 2009: 132.

\section{Material examined}

CHINA: Gansu Prov.: 3 $え$ ๙ , Dongliugou, Sunan, 2500 m a.s.l., 8 Aug. 2007, J.B. Liang leg. (SCAU). -

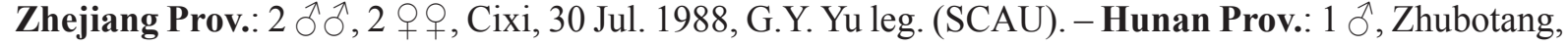

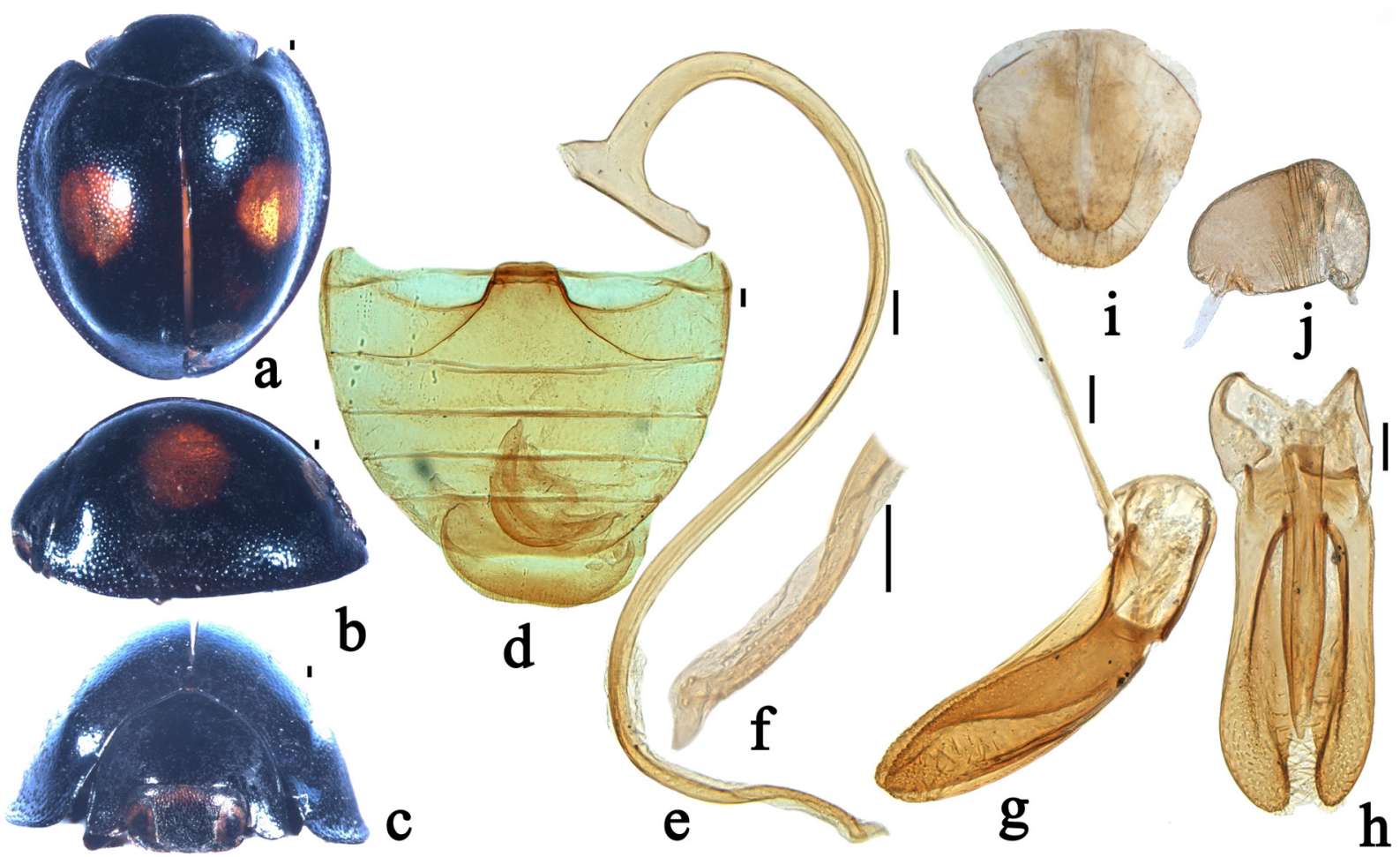

Fig. 12. Chilocorus shirozui Sasaji, 1968. a. Dorsal view. b. Lateral view. c. Frontal view. d. Abdomen. e. Penis. f. Apex of penis. g. Tegmen, lateral view. h. Tegmen, ventral view. i-j. Female genitalia. i. Ovipositor. j. Spermatheca. Scale bars: $0.1 \mathrm{~mm}$. (NCHU) 
Yiyang, 18 Aug. 2001, Z.Q. Peng leg. (SCAU); 1 đ̆, Horticultural institute, Changsha, 1984, collector unknown (SCAU). - Guangdong Prov.: 1 \%, Huidong, 11 May 1987, X.F. Pang leg. (SCAU). Shandong Prov.: 1 specimen, 1958, collector unknown (SCAU). - Sichuan Prov.: 2 specimens, Oct. 1978, collector unknown (SCAU); 3 specimens, Beita, Oct. 1978, collector unknown (SCAU). Guizhou Prov.: 2 ổ, Leigongshan, 30 Jul. 1997, Z.Q. Peng leg. (SCAU); 6 specimens, Huaxi, 18 Aug. 1987, X.F. Pang leg. (SCAU).

\section{Distribution}

China (Zhejiang, Fujian, Shandong, Hubei, Hunan, Sichuan, Guizhou, Guangdong, Guangxi, Gansu) (Fig. 22).

Chilocorus strenotubus Li \& Wang sp. nov. urn:Isid:zoobank.org:act:9258A913-6BF6-419B-812E-C80707513E96

Figs 14, 22

\section{Diagnosis}

This species is similar to C. kuwanae Silvestri, 1909 in the shape of the body, but can be distinguished from it by the male genitalia: penis strongly stout (Fig. 14e); tegmen strongly stout with penis guide subtriangular, widest at base, gradually converging apically to blunted tip in ventral view (Fig. 14h).

\section{Etymology}

The specific epithet is derived from Latin and refers to the stout penis.

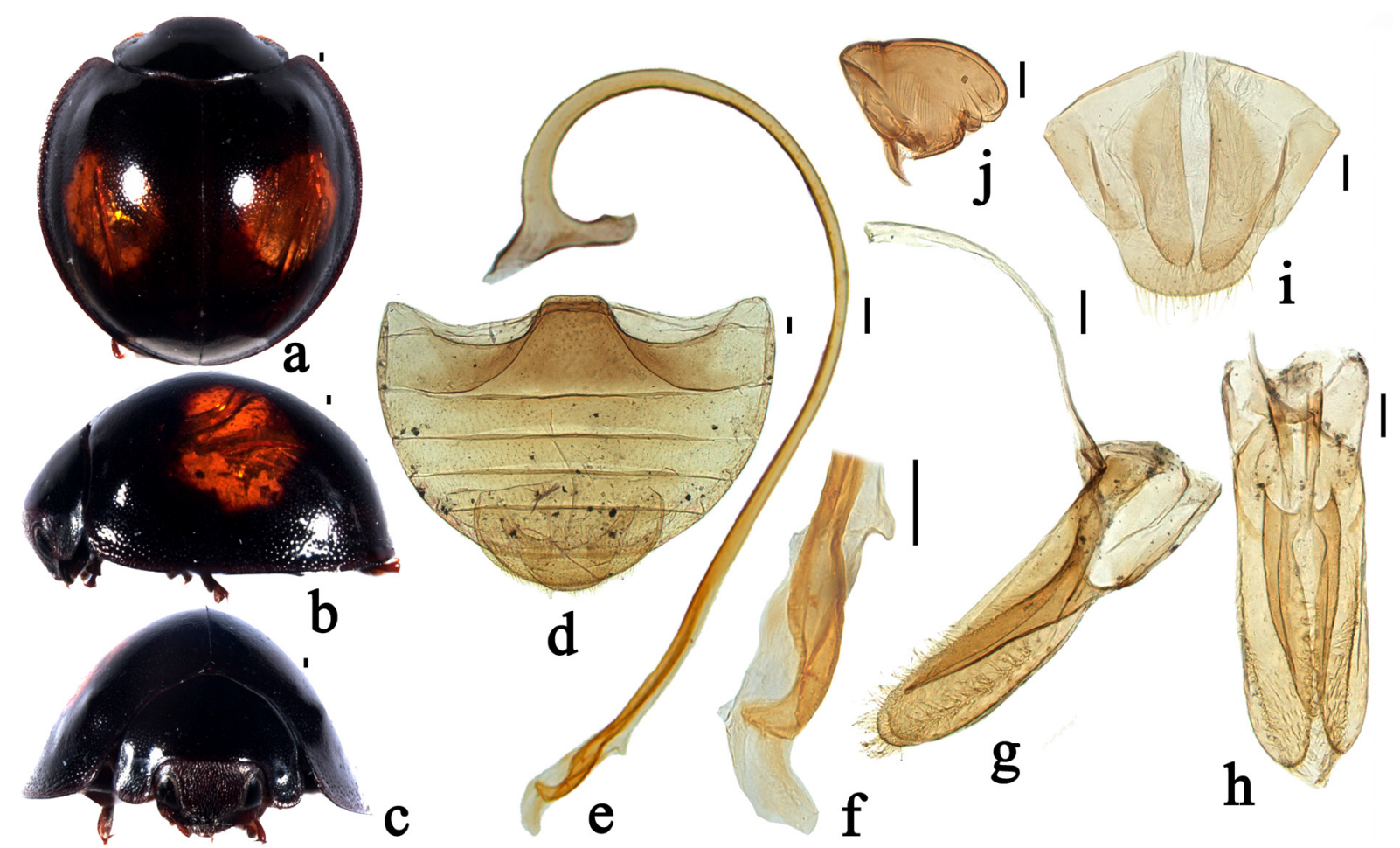

Fig. 13. Chilocorus hupehanus Miyatake, 1970. a. Dorsal view. b. Lateral view. c. Frontal view. d. Abdomen. e. Penis. f. Apex of penis. g. Tegmen, lateral view. h. Tegmen, ventral view. i-j. Female genitalia. i. Ovipositor. j. Spermatheca. Scale bars: $0.1 \mathrm{~mm}$. (SCAU) 


\section{Type material}

\section{Holotype}

CHINA: Ô, Inner Mongolia, Youyizhongqi, Keerqin, 19 Aug. 2009, S.X. Ren leg. (SCAU (E) 11037).

\section{Description (male)}

Measurements. TL: $3.41 \mathrm{~mm}$, TW: 2.93 mm, TH: $1.77 \mathrm{~mm}$, TL/TW: 1.16, PL/PW: 0.51, EL/EW: 1.02.

BoDy. Roundish, strongly convex. Head, antenna and mouthparts brown-black, sparsely covered with short, greyish pubescence. Pronotum and scutellum black. Elytra black with a pair of oblong, orange spots, situated at centre, about $1 / 2$ elytra width (Fig. 14a-c). Underside black except abdomen brown, sparsely covered with short, greyish pubescence. Head relatively large, $0.60 \times$ as wide as pronotum, punctures on frons large and densely distributed, 1.0-2.0 diameters apart, surface polished between punctures. Eyes subtriangular, densely faceted, interocular distance $0.55 \times$ as wide as head (Fig. 14c). Pronotum $0.59 \times$ as wide as elytra, pronotal punctures moderately large and sparsely distributed, smaller than those on head, 2.0-3.5 diameters apart, surface polished between punctures. Punctures on elytra fine and sparsely distributed, 3.0-4.0 diameters apart, smaller than those on pronotum. Prosternal process moderate broad, slightly expanded to apex. Abdominal postcoxal lines incomplete, reaching posterior margin of abdominal ventrite 1 and running along posterior margin and almost reaching lateral margin. Posterior margin of male abdominal ventrite 5 truncate and 6 broadly rounded (Fig. 14d).

Male genitalia. Penis stout and simple, penis capsule with short outer arms and long inner arms, apex of penis truncate and membranous around at $1 / 3$ to apex (Fig. 14e-f). Tegmen distinctly stout with penis guide subtriangular, widest at base, gradually converging to blunt apex in ventral view and widest at base, gradually converging to blunt apex in lateral view. Parameres distinctly longer than penis guide
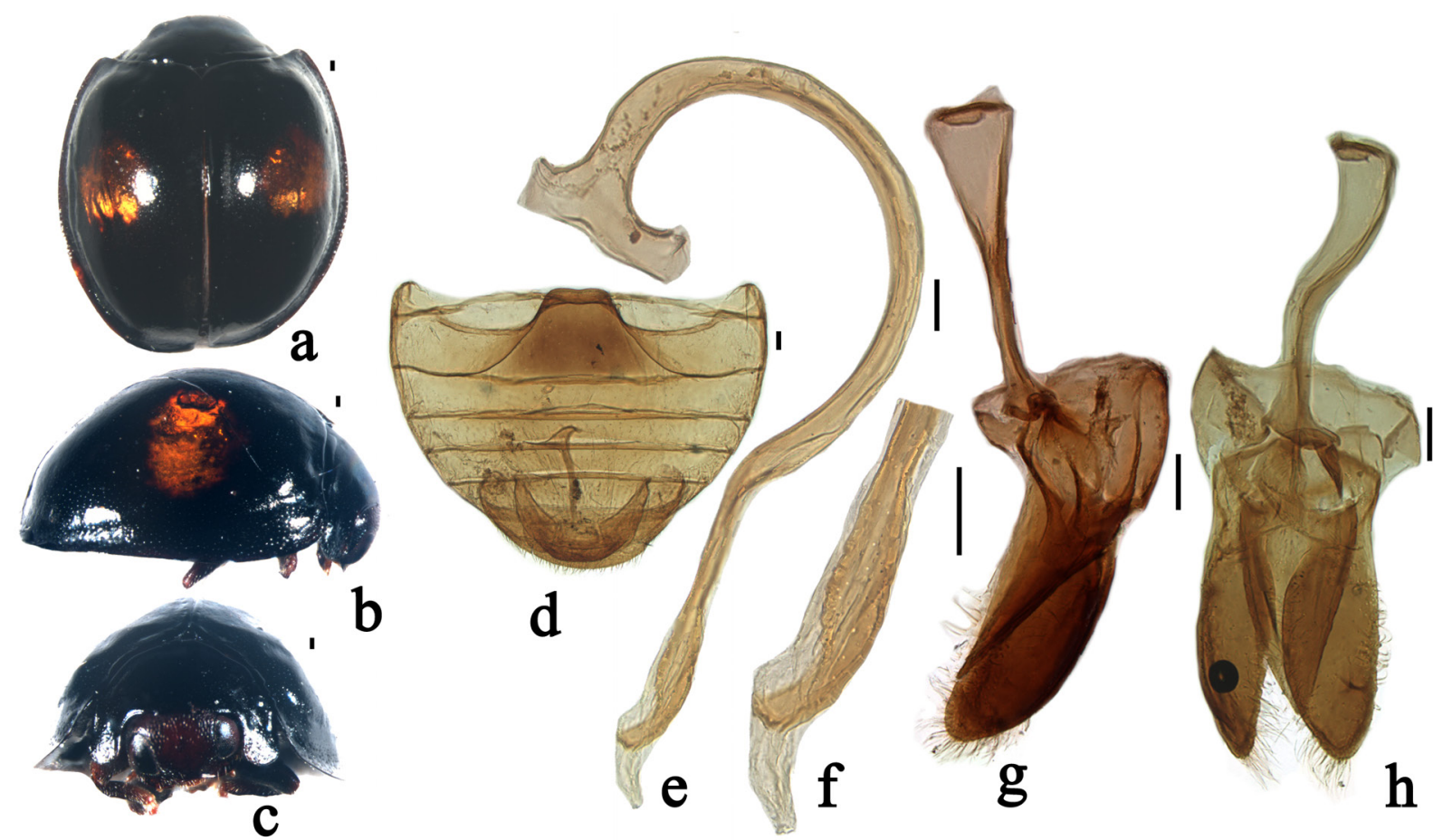

Fig. 14. Chilocorus strenotubus Li \& Wang sp. nov., holotype a. Dorsal view. b. Lateral view. c. Frontal view. d. Abdomen. e. Penis. f. Apex of penis. g. Tegmen, lateral view. h. Tegmen, ventral view. Scale bars: $0.1 \mathrm{~mm}$. (SCAU (E) 11037) 
with densely covered with long setae at the inner sides and distal end with a group of long setae in lateral view (Fig. 14g-h).

\section{Distribution}

China (Inner Mongolia) (Fig. 22).

Chilocorus kuwanae Silvestri, 1909

Figs 15, 22

Chilocorus kuwanae Silvestri, 1909: 126.

Chilocorus similis Lewis, 1896: 31.

Chilocorus similis var. japonicus Sicard, 1907a: 211.

Chilocorus renipustulatus Lewis, 1873: 56.

Chilocorus kuwanae - Korschefsky 1932: 563. - Mader 1955a: 78; 1955b: 776. - Kamiya 1959: 100. — Liu 1963: 77. — Nagaraja \& Hussainy 1967: 251. — Miyatake 1970: 327. — Sasaji 1971: 226. — Pang \& Mao 1979: 87. — Gordon 1985: 652. — Jing 1992: 567. — Cao et al. 1992: 160. — Pang et al. 2002: 319; 2004: 28. — Kováŕ 2007: 593. — Ren et al. 2009:132.

\section{Material examined}

CHINA: Inner Mongolia: 2 $\partial^{\lambda}$, Xiangchizi, Gulaben, 1861-2283 m, 7 Aug. 2010, C.W. Li leg. (SCAU); 1 ․ Honghuaerji, Ewenkeqi, 720 m a.s.l., 22 Aug. 2009, S.X. Ren leg. (SCAU); 1 +, Youyizhongqi, Keerqin, 19 Aug. 2009, S.X. Ren leg. (SCAU). - Xinjiang Uygur Autonomous Region: 1 , , Shanshan, 14 Aug. 2008, S.X. Ren leg. (SCAU); 1 †, Bole, 16 Aug. 2008, S.X. Ren leg. (SCAU). - Ningxia

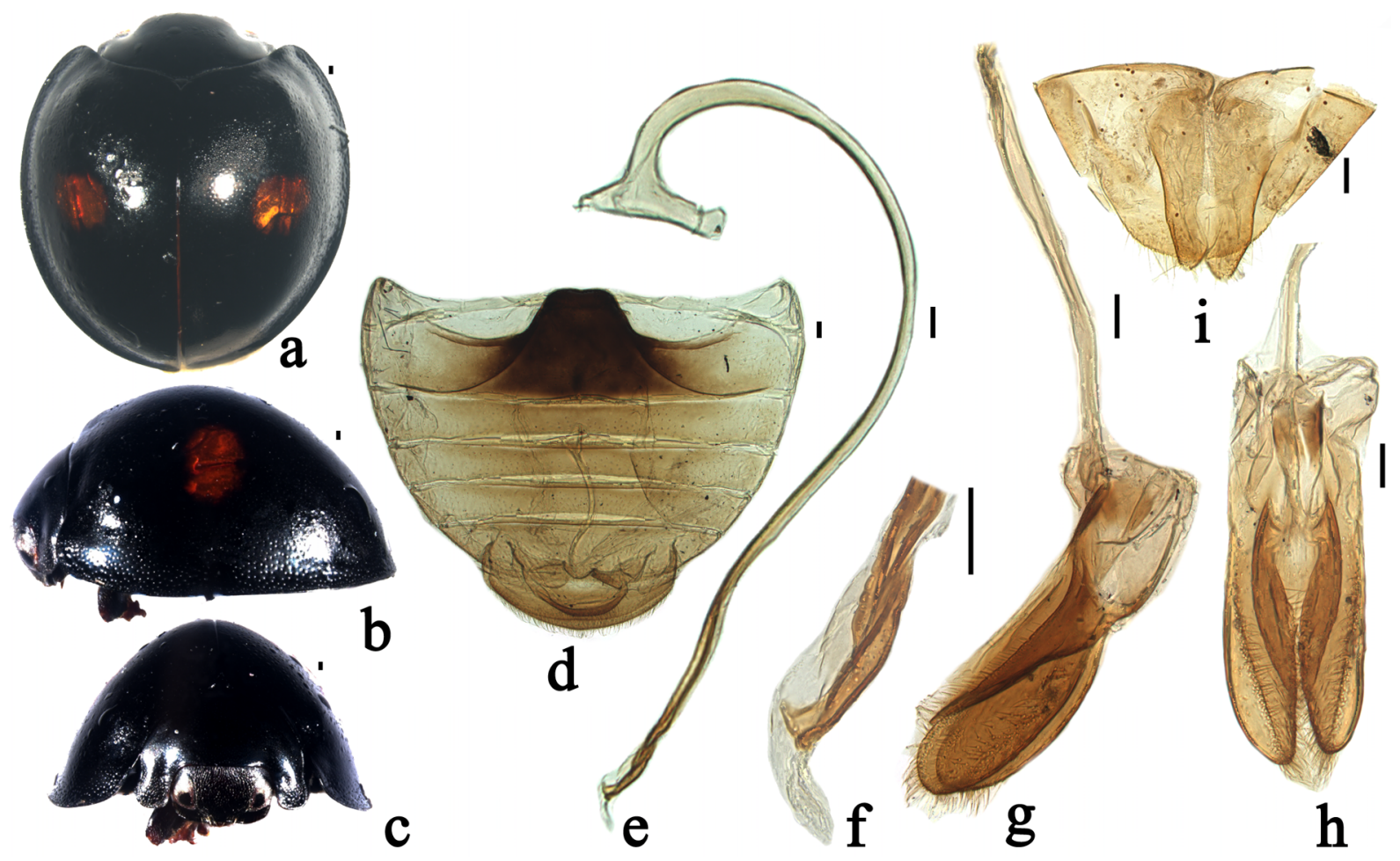

Fig. 15. Chilocorus kuwanae Silvestri, 1909. a. Dorsal view. b. Lateral view. c. Frontal view. d. Abdomen. e. Penis. f. Apex of penis. g. Tegmen, lateral view. h. Tegmen, ventral view. i. ovipositor. Scale bars: $0.1 \mathrm{~mm}$. (SCAU) 
Hui Autonomous Region: 4 q $q$, Suyukou, Yinchuan, 1950 m a.s.1., 12 Aug. 2009, X.M. Wang leg. (SCAU). - Henan Prov.: 1 +, Sanmenxia, 8 Aug. 2008, S.X. Ren leg. (SCAU). - Hubei Prov.: 1 ○, Tiantangzhai, Yingshanxian, 30 Jun. 2014, L.Z. Huo leg. (SCAU); 1 ${ }^{\lambda}$, Qiangtaiguan, Luoyangxian, 2 Jul. 2014, L.Z. Huo leg. (SCAU). - Guangdong Prov.: 1 đ̃, Shenzhen, 19 May 1988, S.P. Shen leg. (SCAU); 6 우, Huidong, 11 May 1988, X.F. Pang leg. (SCAU). - Hunan Prov.: 1 §, Shaowu, 24 Aug. 1984, X.F. Pang leg. (SCAU); 1 \%, Changsha, 1984, collector unknown (SCAU).

\section{Distribution}

China (Beijing, Hebei, Shanxi, Liaoning, Jilin, Heinongiang, Shanghai, Jiangsu, Zhejiang, Anhui, Fujian, Jiangxi, Shandong, Henan, Hubei, Hunan, Guangdong, Guangxi, Sichuan, Guizhou, Yunnan, Shannxi, Gansu, Ningxia, Hong Kong, Inner Mongolia, Xinjiang) (Fig. 22), Japan, North Korea. Introduced to America.

Chilocorus esakii Kamiya, 1959

Figs 16, 22

Chilocorus esakii Kamiya, 1959: 102.

Chilocorus esakii - Sasaji 1971: 227. — Pang \& Mao 1979: 85. — Pang et al. 2004: 28. — Kováŕ 2007: 593.

\section{Material examined}

CHINA: Inner Mongolia: $2 \widehat{\delta} \widehat{\partial}, 2$ + $q$, Guyang, Baotou, $1300 \mathrm{~m}$ a.s.1., 16 Aug. 2009, S.X. Ren

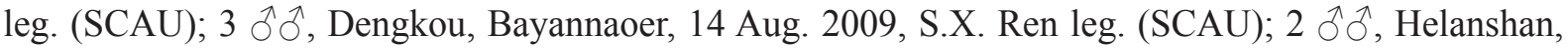

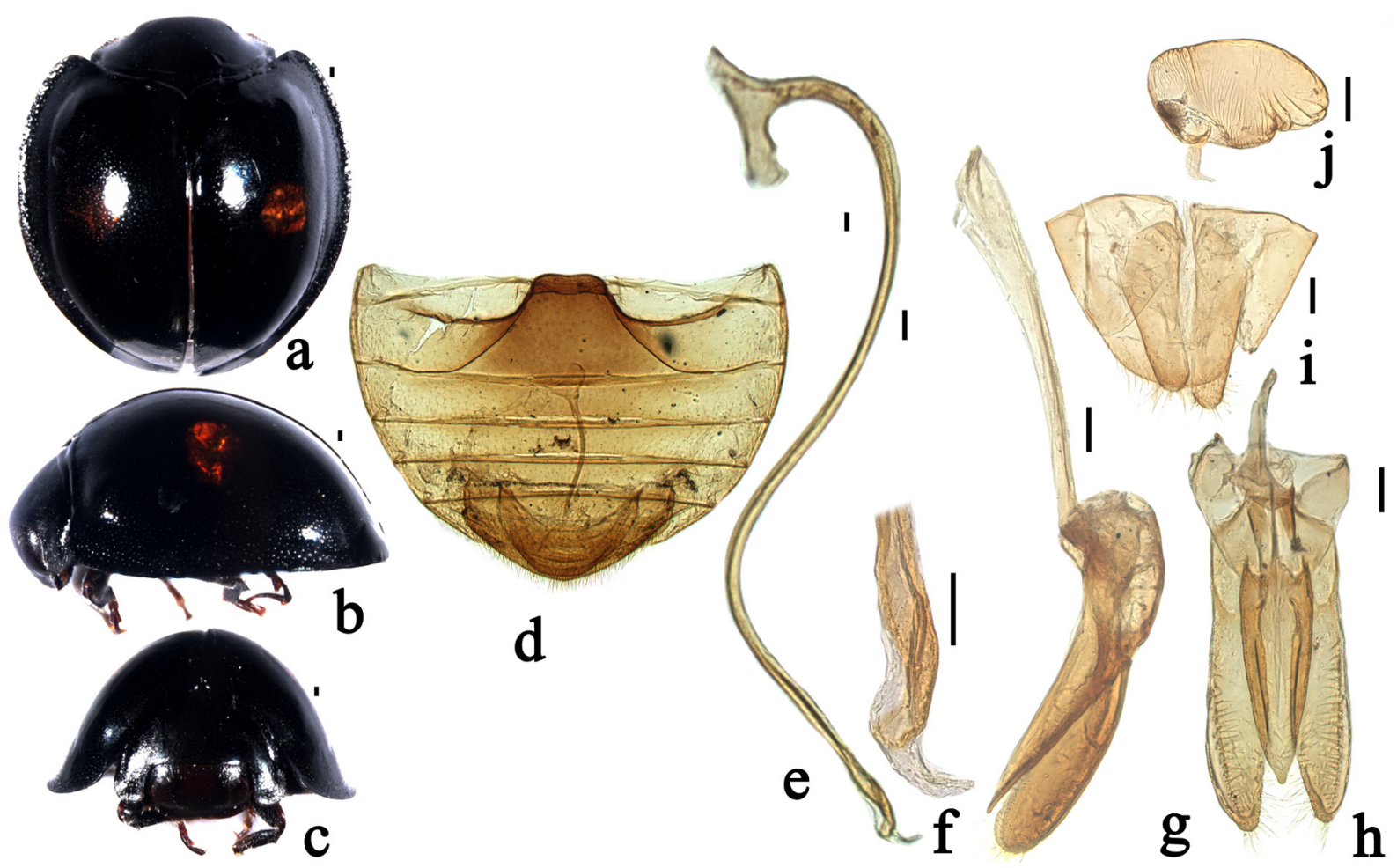

Fig. 16. Chilocorus esakii Kamiya, 1959. a. Dorsal view. b. Lateral view. c. Frontal view. d. Abdomen. e. Penis. f. Apex of penis. g. Tegmen, lateral view. h. Tegmen, ventral view. i-j. Female genitalia. i. Ovipositor. j. Spermatheca. Scale bars: $0.1 \mathrm{~mm}$. (SCAU) 
Alashanzuoqi, 14 Aug. 2009, S.X. Ren leg. (SCAU); 1 đ, Youyizhongqi, Keerqin, 19 Aug. 2009, S.X. Ren leg. (SCAU); 1 đ̊, 2 우, Xiangchizi, Gulaben, 1861-2283m, 7 Aug. 2010, C.W. Li leg. (SCAU). Anhui Prov.: 1 , , Hengdu, Shitai, 230 m a.s.l., 20 Sep. 2010, X.M. Wang leg. (SCAU). - Zhejiang Prov.: 1 §, 7 우, Cixi, 30 Jul. 1988, G.Y. Yu leg. (SCAU). - Hunan Prov.: 1 § 2 우, Horticultural institute, 1984, collector unknown (SCAU); 1 \&, Shaowu, 24 Aug. 1984, X.F. Pang leg. (SCAU). - Guangdong Prov.: 2 q $q$, Huidong, 11 May 1988, X.F. Pang and X.L. Tong leg. (SCAU); 1 , Huidong, 13 Jun. 1988, X.F. Pang leg. (SCAU). - Henan Prov.: 1 ô, Jigong mountain, 10 Jul. 1997, Z.Q. Peng leg. (SCAU); 1 , , Boerdeng part, Xinyang, 216 m a.s.1., 5 Jul. 2009, X.M. Wang leg. (SCAU). - Jiangxi Prov.: 1 ○, Taihe, 12 Aug. 2004, X.M. Wang leg. (SCAU). - Guizhou Prov.: 1 §̂, Qingzhen, 20 Jul. 1994, M.Y. Tian leg. (SCAU). - Fujian Prov.: 1 ऽ, Tongmu, 3 Aug. 1983, K.C. Zhang leg. (SCAU). - Guangxi

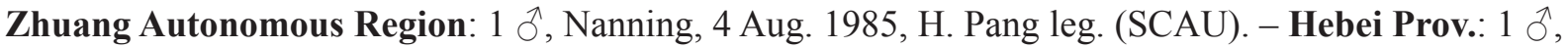
Baoding, 24 Nov. 1960, collector unknown (SCAU).

\section{Distribution}

China (Inner Mongolia, Hebei, Henan, Shanxi, Shanghai, Liaoning, Shandong, Anhui, Jiangxi, Zhejiang, Hunan, Fujian, Guangdong, Sichuan, Guizhou, Guangxi) (Fig. 22), Japan.

\section{Chilocorus alishanus Sasaji, 1968}

Figs 17, 22

Chilocorus alishanus Sasaji, 1968a: 20.

Chilocorus alishanus - Pang \& Mao 1979: 89. — Cao et al. 1992: 162. — Pang et al. 2004: 27. — Kovář 2007: 593. — Yu 2011: 67.
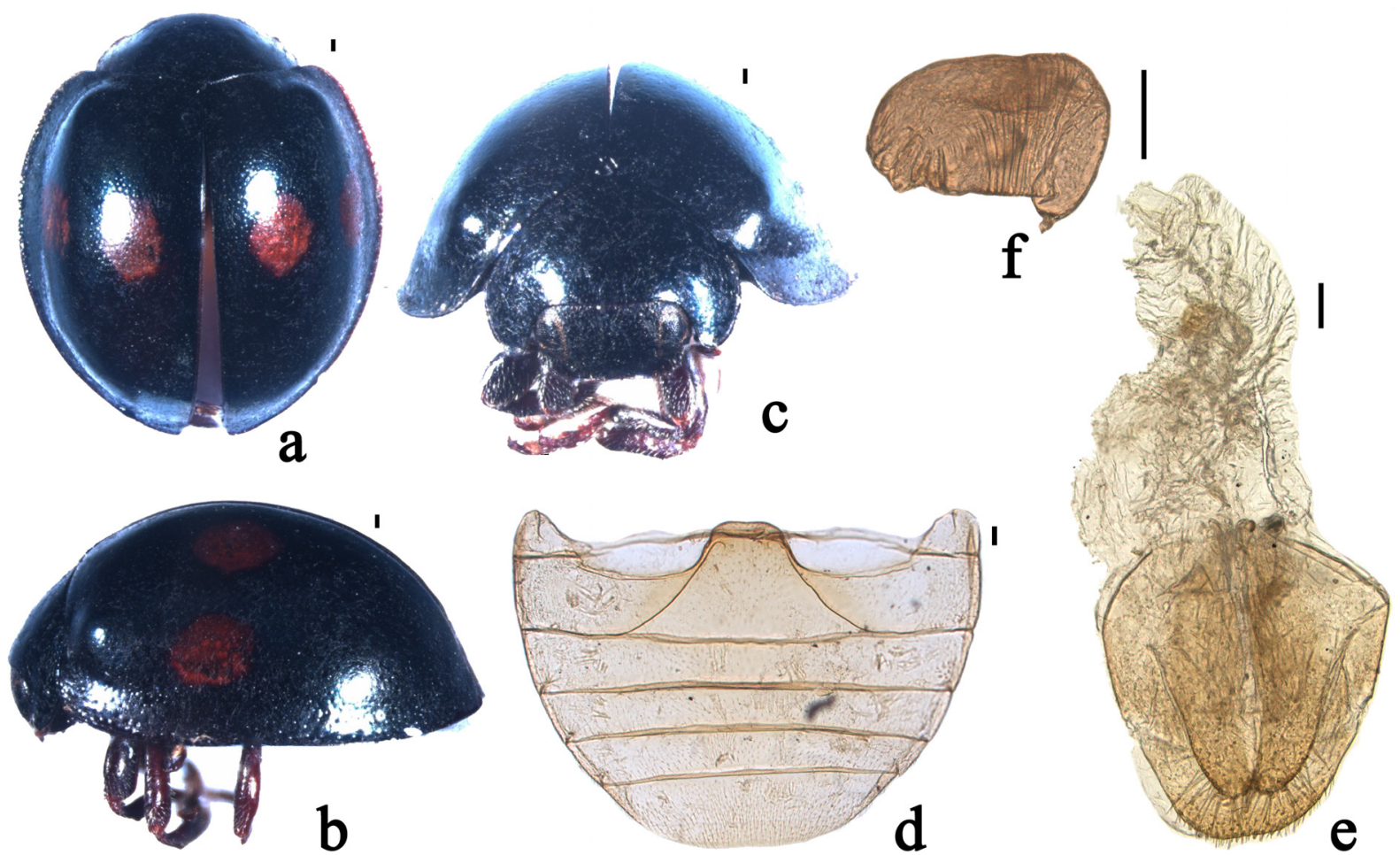

Fig. 17. Chilocorus alishanus Sasaji, 1968. a. Dorsal view. b. Lateral view. c. Frontal view. d. Abdomen. e-f. Female genitalia. e. Ovipositor. f. Spermatheca. Scale bars: $0.1 \mathrm{~mm}$. (NCHU) 


\section{Material examined}

CHINA: Taiwan Prov.: 4 우, Ali Mountain, 1 Jun. 1972, collector unknown (NCHU).

\section{Distribution}

China (Yunnan, Taiwan) (Fig. 22).

\section{Chilocorus bijugus Mulsant, 1853}

Figs 18, 22

Chilocorus bijugus Mulsant, 1853: 61.

Chilocorus infernalis Mulsant, 1853: 189.

Chilocorus bijugus infernalis Korschefsky, 1932: 242.

Chilocorus bijugus - Crotch 1874: 183. — Korschefsky 1932: 242. — Kapur 1956: 259. — Nagaraja \&

Hussainy 1967: 249. — Miyatake 1970: 324; 1985: 12. — Pang \& Mao 1979: 90. — Jing 1992:

566. — Cao et al. 1992: 162. — Pang et al. 2004: 27. — Kovář 2007: 593. — Ren et al. 2009: 126.

Chilocorus infernalis - Crotch 1874: 183.

Chilocorus renipustulatus - Liu 1963: 79.

\section{Material examined}

CHINA: Yunnan Prov.: 3 $\widehat{\jmath}$, Shanggeri-La, 3000 m a.s.1., 3 Sep. 2005, X.M. Wang leg. (SCAU); 1 †, Fenshuiling, Jinping, 2000 m a.s.1., 18 May 2009, S.X. Ren leg. (SCAU); 1 \%, Part of Xishan, Kunming, 2200 m a.s.1., 22 Aug. 2013, X.S. Chen leg. (SCAU). - Sichuan Prov.: 1 q, Panzhihua, 1400 m a.s.1., 16 Sep. 2007, X.M. Wang leg. (SCAU); 1 §̃, Fengyongzhai, Baoxing, 1560 m a.s.l.,

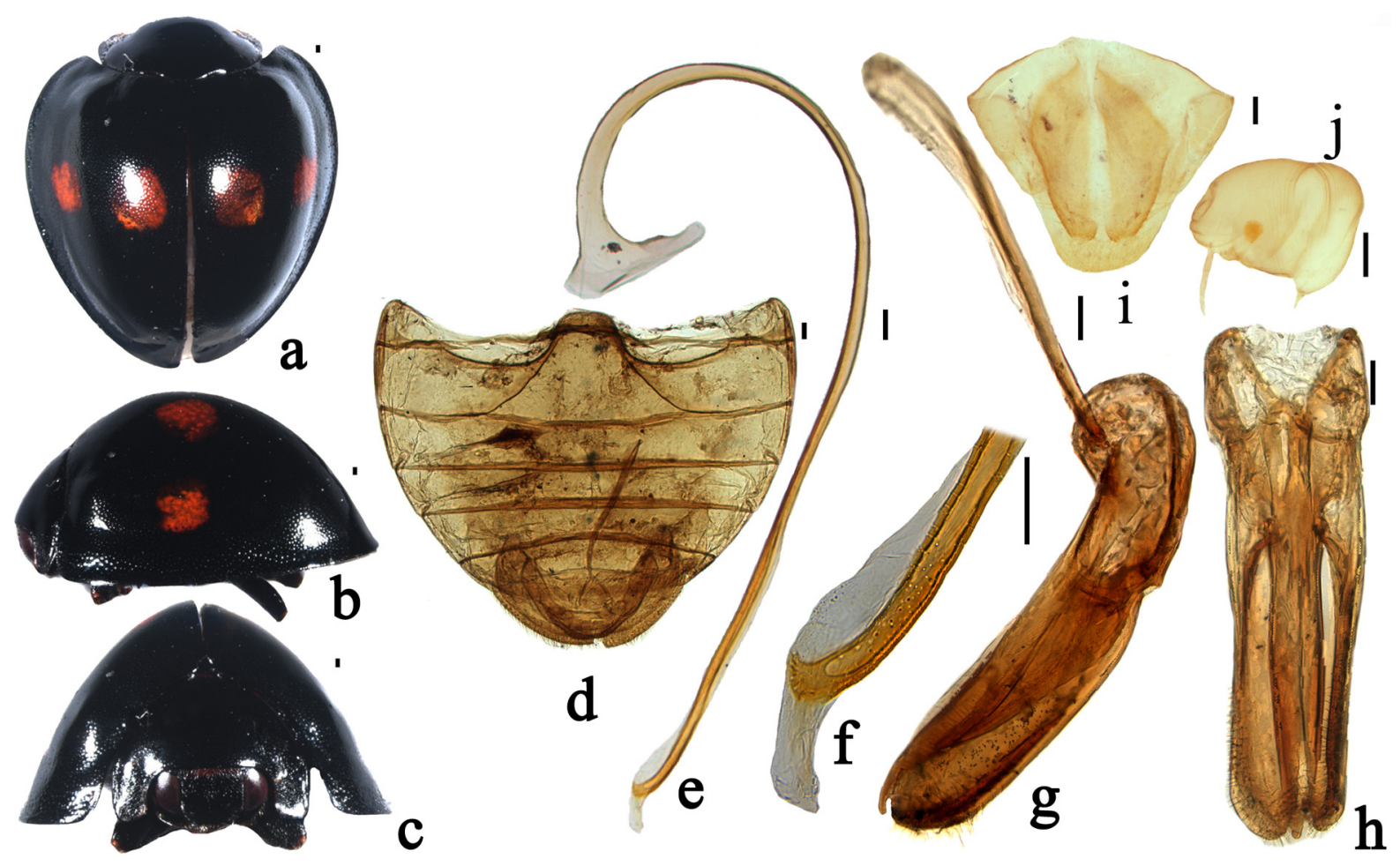

Fig. 18. Chilocorus bijugus Mulsant, 1853. a. Dorsal view. b. Lateral view. c. Frontal view. d. Abdomen. e. Penis. f. Apex of penis. g. Tegmen, lateral view. h. Tegmen, ventral view. i-j. Female genitalia. i. Ovipositor. j. Spermatheca. Scale bars: $0.1 \mathrm{~mm}$. (SCAU) 
2-3 Oct. 2007, J.Y. Hao leg. (SCAU). - Guizhou Prov.: 6 specimens, Huaxi Guiyang, 12 Aug. 1987, X.F. Pang leg. (SCAU); 4 specimens, Guiyang arboretum, 17-19 Aug. 1987, X.F. Pang XF and B.C. Huang leg. (SCAU); 36 specimens, Qingzhen, 20 Jul. 1994, M.Y. Tian leg. (SCAU); 11 specimens, Ximu, Guiyang, 31 Jul. 1994, M.Y. Tian leg. (SCAU); 2 specimens, Huaxi Guiyang, 3 Aug. 1994,

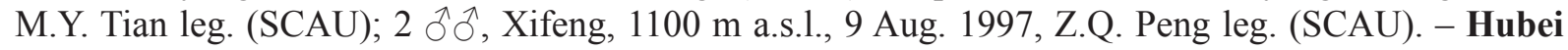
Prov.: 1 , Pingqian, Shennongjia, 1600 m a.s.l., 27 Jul. 2007, X.M. Wang leg. (SCAU); 1 , Yinyu River, Shennongjia, 1700 m a.s.l., 31 Jul. 2007, X.M. Wang leg. (SCAU); 1 ô, Laojun Mountain, Shennongjia, $1230 \mathrm{~m}$ a.s.l., 5 Aug. 2007, X.M. Wang leg. (SCAU). - Tibet: 1 §, Mangkang, $2300 \mathrm{~m}$ a.s.1., 10 Oct. 2007, X.M. Wang leg. (SCAU); 1 đ, Mangkang, 3300 m a.s.1., 18 Sep. 2011, L.Z. Huo leg. (SCAU); 1 §̂, Yigong, Bomi, 2100 m a.s.1., 18 Oct. 2011, L.Z. Huo leg. (SCAU); 1 O, Milin, Paizhen, 2950 m a.s.1., 21 Oct. 2011, L.Z. Huo leg. (SCAU).

\section{Distribution}

China (Jiangsu, Hubei, Sichuan, Guizhou, Yunnan, Tibet, Gansu) (Fig. 22), Japan, India, Nepal, Pakistan, Palaearctic.

Chilocorus bipustulatus (Linnaeus, 1758)

Figs 19, 22

Coccinella bipustulata Linnaeus, 1758: 367.

Chilocorus bipustulatus - Crotch 1874: 185. — Jakobson 1916: 990. — Korschefsky 1932: 239. — Bielawski 1984: 369. — Pang \& Mao 1979: 88. — Gordon 1985: 654. — Poorani 2002: 311. — Pang et al. 2004: 27. — Kovář 2007: 593. — Ren et al. 2009: 128.

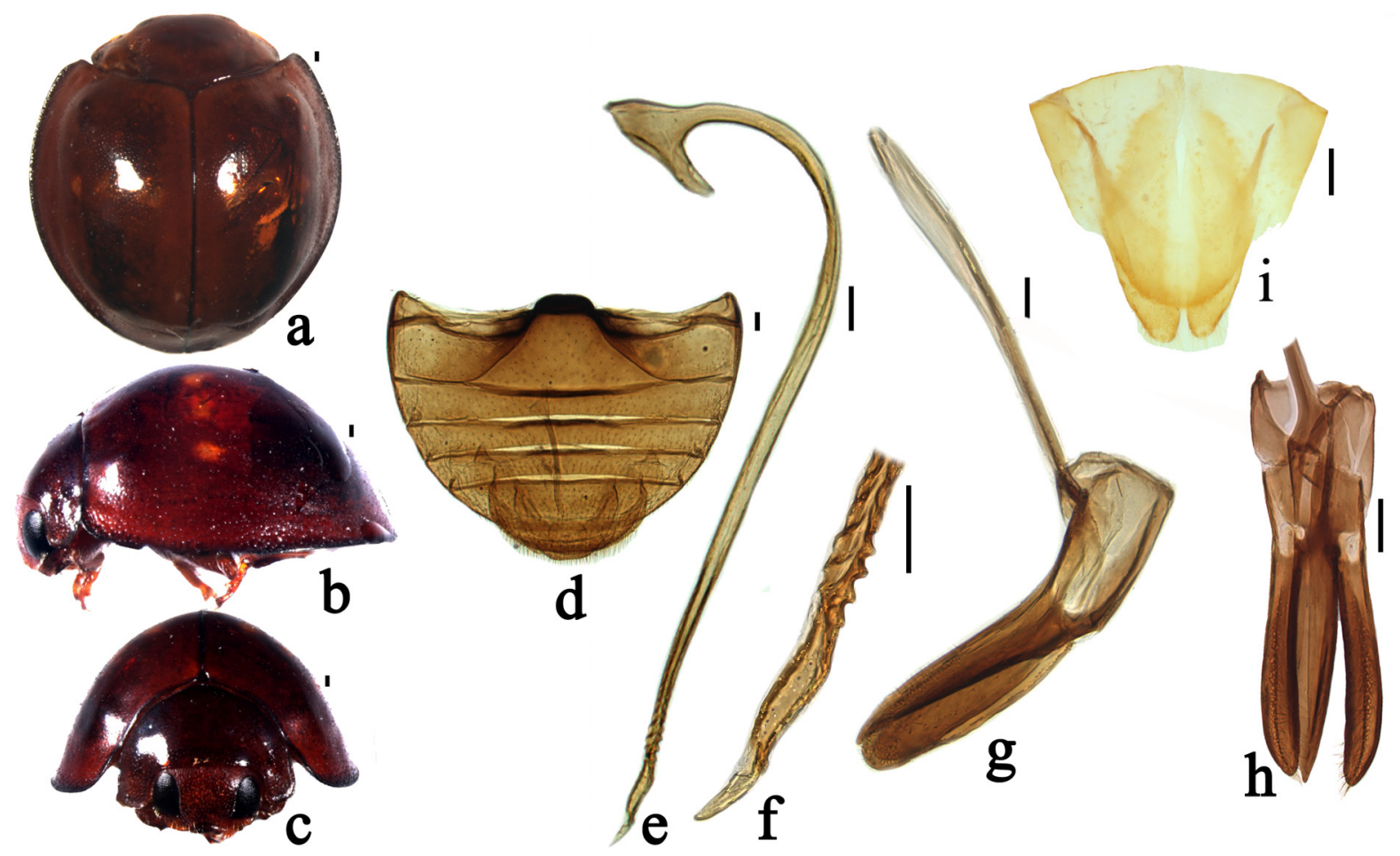

Fig. 19. Chilocorus bipustulatus (Linnaeus, 1758). a. Dorsal view. b. Lateral view. c. Frontal view. d. Abdomen. e. Penis. f. Apex of penis. g. Tegmen, lateral view. h. Tegmen, ventral view. i. ovipositor. Scale bars: $0.1 \mathrm{~mm}$. (SCAU) 


\section{Material examined}

CHINA: Xinjiang Uygur Autonomous Region: 5 $\widehat{\partial}, 2$ q $q$, Bole, 1000 m a.s.1., 16 Aug. 2008, S.X. Ren leg. (SCAU); 1 Oे, Tianchi, Fukang, 550 m a.s.l., 5 Sep. 2008, X.M. Wang leg. (SCAU); 1 ㅇ, Shanshan, 14 Aug. 2008, S.X. Ren leg. (SCAU); 1 ðે, Qiaxi, Gongliu, 1430 m a.s.1., 19 Aug. 2008, S.X. Ren leg. (SCAU); 1 ठ, Yining, 950 m a.s.1., 18 Aug. 2008, S.X. Ren leg. (SCAU).

\section{Distribution}

China (Xinjiang) (Fig. 22), Europe, Middle East, Central Asia, North Africa.

Chilocorus geminus Zaslavskij, 1962

Figs 20, 22

Chilocorus geminus Zaslavskij, 1962: 398.

Chilocorus geminus - Bielawski 1975: 254; 1984: 370. — Pang \& Mao 1979: 88. — Pang et al. 2004: 28. — Kovář 2007: 593. — Ren et al. 2009: 130.

\section{Material examined}

CHINA: Xinjiang Uygur Autonomous Region: 1 đึ, Shanshan, 400 m a.s.1., 14 Aug. 2008, S.X. Ren

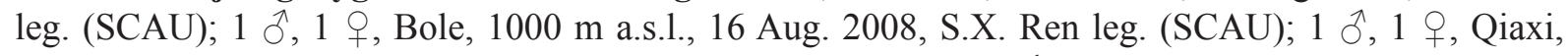
Gongliu, 1430 m a.s.1., 19 Aug. 2008, X.M. Wang leg. (SCAU); 1 §, Hejing or Bohu, $1100 \mathrm{~m}$ a.s.1., 21

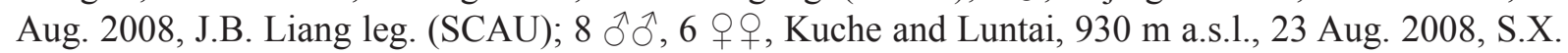

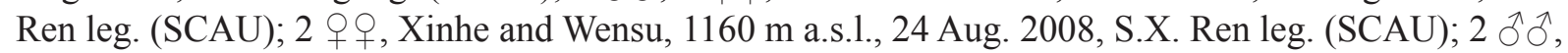

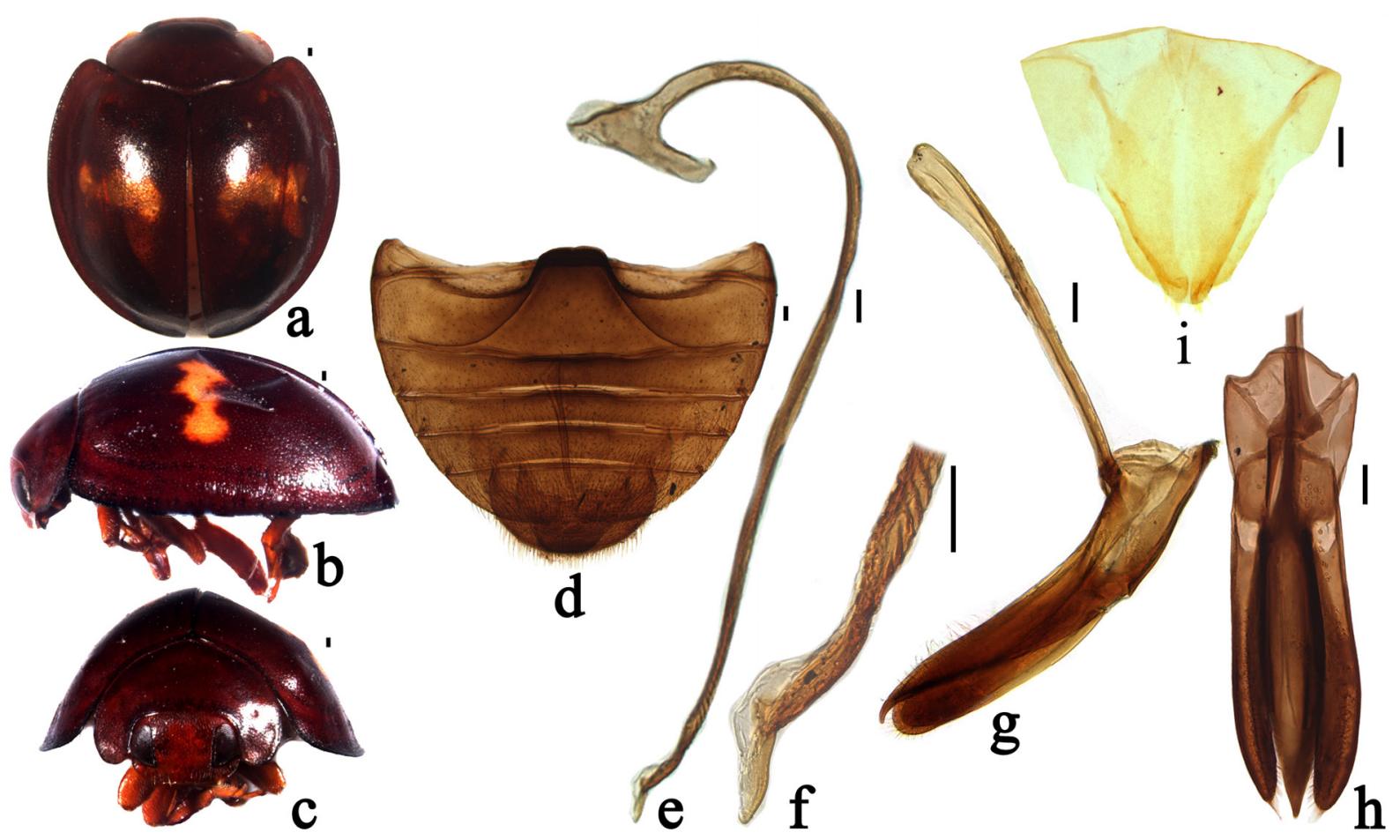

Fig. 20. Chilocorus geminus Zaslavskij, 1962. a. Dorsal view. b. Lateral view. c. Frontal view. d. Abdomen. e. Penis. f. Apex of penis. g. Tegmen, lateral view. h. Tegmen, ventral view. i. ovipositor. Scale bars: $0.1 \mathrm{~mm}$. (SCAU) 
1 , Awati, 1070 m a.s.1., 25 Aug. 2008, S.X. Ren leg. (SCAU); 1 q, Akesu, 1000 m a.s.1., 27 Aug. 2008, X.M. Wang leg. (SCAU).

\section{Distribution}

China (Gansu, Xinjiang) (Fig. 22), Mongolia, Uzbekistan, Turkey, Central Asia.

\section{The world checklist of Chilocorus Leach, 1815}

C. adustus Weise, 1898a: 119. Gabon, Cameroon, West Africa.

C. alishanus Sasaji, 1968a: 20. China.

C. amamensis Kamiya, 1959: 103. Japan.

C. angolensis Crotch, 1874: 186. Angola, Central \& southern Africa.

C. bennigseni Weise, 1900: 128. Tanzania, Central East Africa.

C. bijugus Mulsant, 1853: 61. China, Japan, India, Nepal, Pakistan, Palaearctic.

C. bipustulatus (Linnaeus, 1758): 367. China, Europe, Middle East, Central Asia, North Africa.

C. braeti Weise, 1895b: 154. Himalaya, India.

C. cacti (Linnaeus, 1767): 584. America.

C. calvus Weise, 1898b: 228. Mozambique, South Africa.

C. canariensis Crotch, 1874: 185. Canary Islands.

C. cerberus Mulsant, 1853: 148. Malaysia, Indonesia, Philippines, Sumatra.

C. chalybeatus Gorham, 1892: 24. China.

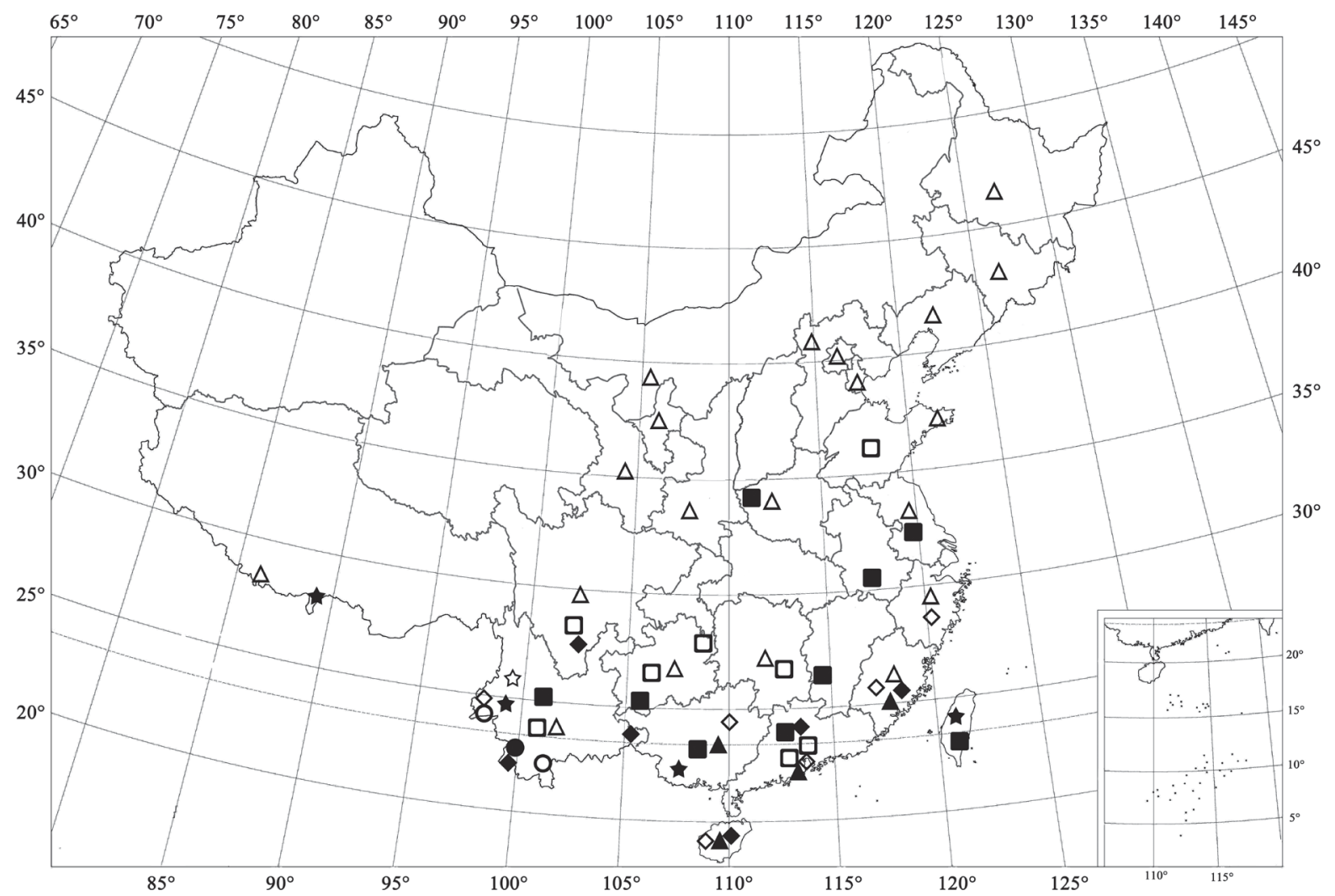

Fig. 21. Distribution map. $(\star)=$ Chilocorus politus Mulsant, 1850; $(\widehat{\varsigma})=C$. yunlongensis Cao \& Xiao, 1984; $(\bullet)=$ C. nigricaeruleus Li \& Wang sp. nov.; $(\circ)=$ C. nigrita (Fabricius, 1798); $(\boldsymbol{\Delta})=$ C. melas Weise, 1898; $(\Delta)=$ C. rubidus Hope, 1831; $(\mathbf{\square})=$ C. chinensis Miyatake, 1970; $(\square)=$ C. rufitarsis Motschulsky, 1853; $(\bullet)=$ C. hauseri Weise, 1895; $(\diamond)=$ C. circumdatus (Gyllenhal, 1808). 
C. chinensis Miyatake, 1970: 322. China.

C. circumdatus (Gyllenhal, 1808): 152. China, Indonesia, India, Sri Lanka. Introduced to Australia, America.

C. coelosimilis Kapur, 1967: 171. India.

C. cooki Casey, 1899: 165. Liberia.

C. crotchi Li \& Ślipiński in Li et al., 2014: 598. Indonesia.

C. cruentatus Gorham, 1901: 413. Zimbabwe.

C. distigma (Klug, 1835): 49. Angola, Ethiopia.

C. dohrni Mulsant, 1850: 456. Guinea, Nigeria, Senegal, Zimbabwe.

C. elegans Mader, 1954: 75. Democratic Republic of the Congo.

C. esakii Kamiya, 1959: 102. Japan.

C. fraternus LeConte, 1860: 70. America.

C. geminus Zaslavskii, 1962: 398. China, Mongolia, Uzbekistan, Turkey, Central Asia.

C. gracilior Booth, 1998: 365. Indonesia.

C. haematocephalus Sicard, 1909: 92. Madagascar.

C. hauseri Weise, 1895a: 135. China, Burma, India, Myanmar.

C. hexacyclus Smith, 1959: 446. Canada.

C. hupehanus Miyatake, 1970: 329. China.

C. insularis Weise, 1906: 208. Madagascar.

C. ishigakensis Kamiya, 1959: 104. Japan.

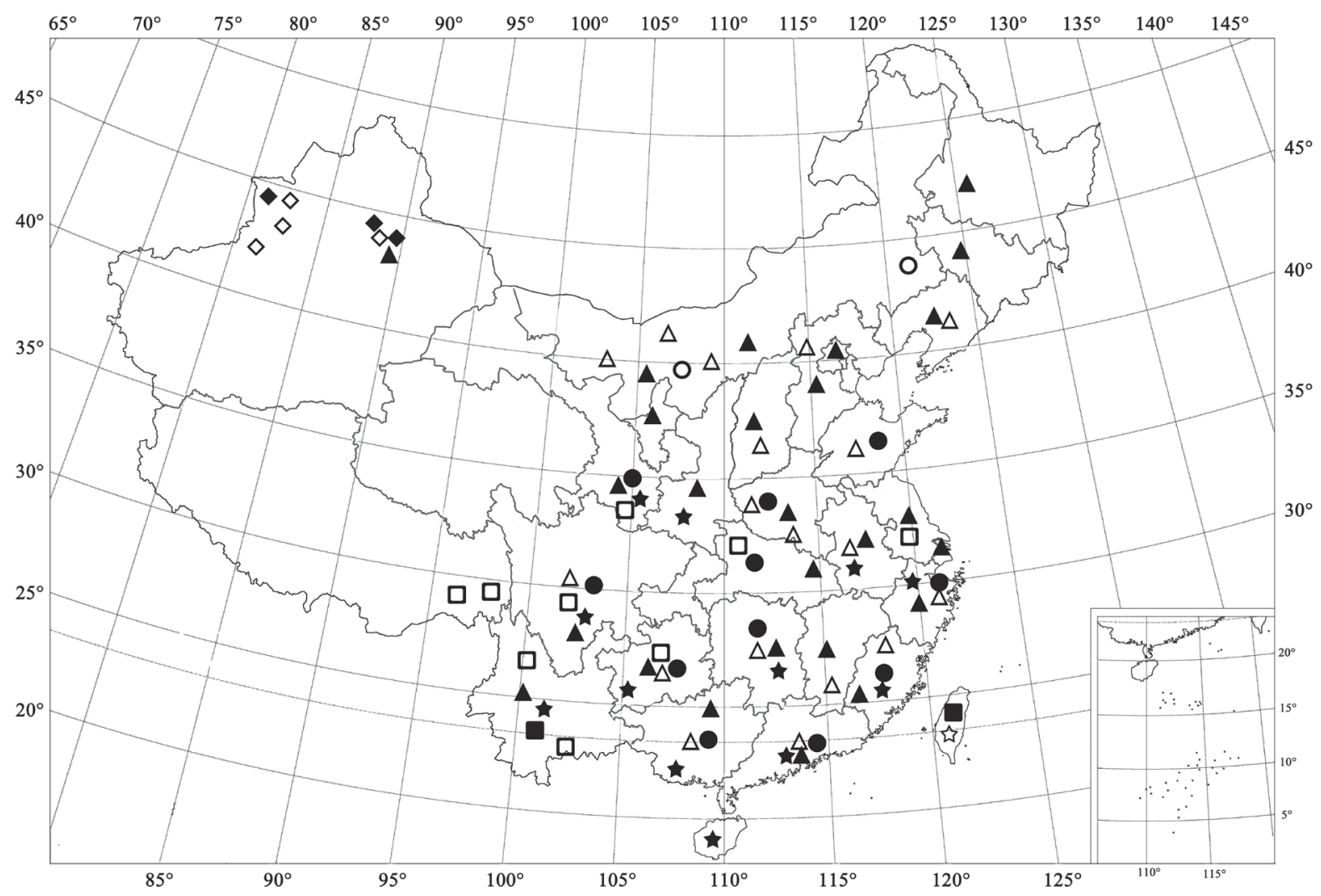

Fig. 22. Distribution map. $(\star)=$ Chilocorus chalybeatus Gorham, 1892; (出 $)=$ C. shirozui Sasaji, 1968; $(\bullet)=$ C. hupehanus Miyatake, 1970; $(0)=$ C. strenotubus Li \& Wang sp. nov.; $(\mathbf{\Delta})=$ C. kuwanae Silvestri, 1909; $(\Delta)=$ C. esakii Kamiya, 1959; $(\mathbf{\square})=$ C. alishanus Sasaji, 1968; $(\square)=$ C. bijugus Mulsant, 1853; $(\diamond)=$ C. bipustulatus (Linnaeus, 1758); $(\diamond)=$ C. geminus Zaslavskij, 1962. 
C. kirgisicus Iablokoff-Khnzorian, 1976: 305. Palaearctic.

C. kuwanae Silvestri, 1909: 126. China, Japan, North Korea, Italy. Introduced to America.

C. malasiae Crotch, 1874: 187. Australia, New Guinea.

C. maculatus Ślipiński \& Giorgi, 2006: 270. Australia.

C. marshalli Gorham, 1901: 414. Angola, Zimbabwe.

C. matsumurai Miyatake, 1985: 13. Nepal.

C. melanophthalmus Mulsant, 1850: 455. Indonesia (Java).

C. melas Weise, 1898b: 229. China, Burma, Thailand, Laos, India, Nepal, Bhutan, Indonesia.

C. metallescens Sicard, 1909: 94. Madagascar.

C. micrus Ślipiński \& Giorgi, 2006: 272. Melville Island (Australia).

C. midas Klug, 1833: 214. Madagascar.

C. nigricaeruleus Li \& Wang sp. nov. China.

C. nigripes Mader, 1954: 73. Tanzania.

C. nigrita (Fabricius, 1798): 79. China, Far East, Indian subcontinent, Oriental region, Australian region, America, Brazil, Africa.

C. orbus Casey, 1899: 105. America.

C. pilosus Sicard, 1920: 212. São Tomé and Príncipe.

C. platycephalus Mulsant, 1850: 464. Cuba.

C. politus Mulsant, 1850: 455. China, Thailand, Laos, India, Nepal, Bhutan, Indonesia.

C. quadriguttatus Weise, 1888: 93. South Africa.

C. quadrimaculatus Weise, 1910: 45. East Africa.

C. reinecki Weise, 1905: 140. South Africa.

C. renipustulatus (Scriba, 1791): 105. Europe.

C. rubidus Hope, 1831: 31. China, Mongolia, Korea, Japan, India, Nepal, Indonesia, Siberia.

C. rubrocinctus Sicard, 1909: 93. Madagascar.

C. rufitarsis Motschulsky, 1853: 50. China, Vietnam.

C. rufithorax Mader, 1954: 78. Democratic Republic of the Congo.

C. schioedtei Mulsant, 1850: 456. Guinea, Sierra Leone, Uganda.

C. semiaeneus Weise, 1891: 284. Tsibodas ('Tzibodes' in Weise 1891, perhaps Cibodas in Indonesia)

C. seminulum Weise, 1910: 46. Kisar Island (Indonesia).

C. sexguttatus Weise, 1912: 115. Angola, Democratic Republic of the Congo, Tanzania.

C. shirozui Sasaji, 1968 a: 22. China.

C. silvestrii Weise, 1913: 221. Democratic Republic of the Congo, Guinea.

C. similis Rossi, 1790: 68. Italy.

C. simoni Sicard, 1907b: 413. South Africa.

C. solitus Wiese, 1899: 62. Mozambique.

C. stigma (Say, 1835): 202. America. Introduced to Hawaii, Australia, Chile, Italy, Mauritania.

C. stillatus Sicard, 1912: 411. Democratic Republic of the Congo.

C. strenotubus Li \& Wang sp. nov. China.

C. subaenescens Weise, 1898b: 227. Tanzania.

C. subindicus Booth, 1998: 365. India, Maldives, Sri Lanka.

C. sublineatus Crotch, 1874: 187. Indonesia.

C. takara Nakane \& Araki, 1959: 52. Japan.

C. tibialis Weise, 1897: 300. Tanzania.

C. tricyclus Smith, 1959: 446. America.

C. trimaculatus Crotch, 1874: 187. Indonesia.

C. tumidus Leng, 1908: 37. America.

C. wahlbergi Mulsant, 1850: 462. Democratic Republic of the Congo, South Africa, Tanzania, Zanzibar.

C. wallacii Crotch, 1874: 187. Indonesia.

C. yunlongensis Cao \& Xiao, 1984: 117. China. 


\section{Discussion}

The morphological characters of the genus Chilocorus are relatively heterogeneous at species level. Chapin (1965) and Miyatake (1970) thought that it should be further subdivided. However, four genera in Chilocorini, Phaenochilus Weise, 1895 distributed in Oriental region, Simmondsius Ahmad \& Ghani,1966 distributed in Pakistan, Anisorcus Crotch, 1874 distributed in Australian region and Egius Mulsant, 1850 distributed in Cuba, are very similar to Chilocorus in morphological characters. A more detailed analysis of the phylogeny of the genera of Chilocorini is needed to reveal the relationships between the five groups and determine whether Chilocorus is a natural group.

\section{Acknowledgements}

We are particularly grateful to Wioletta Tomaszewska (Museum and Institute of Zoology PAS, Warsaw, Poland) and Michael Geiser (Natural History Museum, London, UK) for their kind help during Wenjing Li's visit to the museums. We are grateful to Shaukat Ali (South China Agriculture University, Guangzhou, China) for checking a draft of this paper. This research was supported by the National Natural Science Foundation of China (31501884), Science and Technology Partnership Program, Ministry of Science and Technology of China (KY201402014) and Science and Technology Program of Guangzhou, China (201509010023).

\section{References}

Bielawski R. 1957. Coccinellidae (Coleoptera) von Ceylon. Verhandlungen der naturforschenden Gesellschaft in Basel 68: 72-96.

Bielawski R. 1975. Ergebnisse der zoologischen Forschungen von Dr. Z. Kaszab in der Mongolei, Nr. 352. Coccinellidae V and VI (Coleoptera). Fragmenta Faunistica (Warzawa) 20: 247-271.

Bielawski R. 1984. Coccinellidae (Coleoptera) of Mongolia. Annales Zoologici (Warszawa) 38: 281460. Available from http://rcin.org.pl/Content/58507/WA058_10823_P255-T38_Annal-Zool-Nr-14.pdf [accessed 23 Aug. 2018].

Blackburn T. 1889. Further notes on Australian Coleoptera, with descriptions of new species. Transactions of the Royal Society of South Australia 11: 175-214.

Booth R.G. 1998. A review of the species resembling Chilocorus nigrita (Coleoptera: Coccinellidae): potential agents for biological control. Bulletin of Entomological Research 88 (4): 361-367.

Booth R.G. \& Pope R.D. 1989. A review of the type material of Coccinellidae (Coleoptera) described by F.W. Pope and by E. Mulsant in the Hope Entomological Collections, Oxford. Entomologica Scandinavica 20 (3): 343-370.

Casey T.L. 1899. A revision of the American Coccinellidae. Journal of the New York Entomological Society 7: 71-169.

Cao C.Y. \& Xiao N.N. 1984. New species of Coccinellidae from Yunnan, China. Entomotaxonomia 6: 109-132. [In Chinese.]

Cao C.Y., Pan Y.Z. \& Wang H. 1992. Coccinellidae of Yunnan. Yunnan Science \& Technology Publishing House, Kunming. [In Chinese.]

Chapin E.A. 1965. The genera of the Chilocorini (Coleoptera, Coccinellidae). Bulletin of the Museum of Comparative Zoology, Harvard University 133: 227-271.

Chazeau J., Étienne J. \& Fürsh H. 1974. Les Coccinellidae de l'île de La Réunion (Insecta Coleoptera). Bulletin du Museum national d'Histore naturelle 140: 265-297. 
Chen L.H., Zhang S.Q., Li Y., Liang D., Pang H., Ślipiński A. \& Zhang P. 2017. Genome-wide survey of nuclear protein-coding markers for beetle phylogenetics and their application in resolving both deep and shallow-level divergences. Molecular Ecology Resources 1-17.

https://doi.org/10.1111/1755-0998.12664

Crotch G.R. 1874. A Revision of the Coleopterous Family Coccinellidae. E. W. Department of Agriculture, Govermnent Research Institute, Taihoku 55: 1-359.

Escalona H.E., Zwick A., Li H.S., Li J.H., Wang X.M, Pang H., Hartley D., Jermiin L.S., Nedvěd O., Misof B., Niehuis O., Ślipiński A. \& Tomaszewska W. 2017. Molecular phylogeny reveals food plasticity in the evolution of true ladybird beetles (Coleoptera: Coccinellidae: Coccinellini). BMC Evolutionary Biology 17 (1): 151. https://doi.org/10.1186/s12862-017-1002-3

Fabricius J.C. 1798. Supplementum entomologiae systematicae. Proft and Storch, Copenhagen.

Faldermann F. 1835. Coleopterorum ab ill. Bungio in China boreali, Mongolia et montibus Altaicis collectorum, nee non ab ill. Turczaninoffio et Stschukino e provincia Irkutzk missorum illustrationes. Memoires de l'Académie impériale des Sciences des Saint Petersbourg 2: 337-464.

Giorgi J.A., Vandenberg N.J., Mchugh J.V., Forrester J.A., Ślipiński S.A., Miller K.B., Shapiro L.R. \& Whiting M.F. 2009. The evolution of food preferences in Coccinellidae. Biological Control 51: 215231. https://doi.org/10.1016/j.biocontrol.2009.05.019

Gordon R.D. 1985. The Coccinellidae (Coleoptera) of America north of Mexico. Journal of the New York Entomological Society 93: 1-912.

Gorham H.S. 1892. Coleoptera from Central China and the Korea. The Entomologists 25 (4) (Supplement): $81-85$.

Gorham H.S. 1901. Descriptions of genera and species of Coleoptera from South Africa. Annals and Magazine of Natural History 7: 401-415.

Gyllenhal L. 1808. [New taxa.] In: Schönherr C.J. (ed.) Synonyma Insectorum, oder: Versuch einer Synonymie aller bisher bekannten Insecten; nach Fabricii Systema Eleutheratorum \& c. geordnet. Erster Band. Eleutherata oder Käfer. Zweiter Theil. Sprechus-Cryptocepalus. C.F Marquard, Stockholm.

Hoáng D.N. 1983. Coccinellidae of Vietnam. Part. 2. Department of Science and Technology, Hanoi, 1-159. [In Vietnamese with English summary.]

Hope F.W. 1831. Synopsis of the new species of Nepaul insects in the collection of Major General Hardwicke. In: Gray J.E. (ed.) Zoological Miscellany: 21-32. Treutel, Wurtz and Co, London.

Hu J.F. 1937. Coleoptera-Coccinellidae, Catalogus Insectorum Sinensium, Vol. 3: 553-587. The Fan Memorial Institute of Biology, Peiping. [In Chinese.]

Iablokoff-Khnzorian S.M. 1976. Dva novykh vida zhestkokrylyhk-kokcinellid iz aziatskoy chasti SSSR (Coleoptera, Coccinellidae). Doklady Akademii Nauk Armyanskoy SSR 62: 305-309.

Jakobson G.G. 1916. Die Käfer Russlands und Westeuropas. Ein Handbuch zum Bestimmen der Käfer; 865-1024. Imperial Academy of Sciences, Petrograd.

Jing X. 1992. Coleoptera: Coccinellidae. In: Chen S. (ed.) Insects of the Hengduan Mountains Region: 541-574. Science Press, Beijing. [in Chinese with English summary.]

Kamiya H. 1959. A revision of the tribe Chilocorini of Japan and the Loochoos (Coleoptera: Coccinellidae). Kontyu 27 (2): 99-105. 
Kapur A.P. 1956. Systematic and biological notes on the ladybird beetles predacious on the San Jose scale in Kashmir with description of a new species (Coleoptera: Coccinellidae). Records of the Indian Museum 52 (1954): 257-274.

Kapur A.P. 1967. The Coccinellidae (Coleoptera) of the Andamans. Proceedings of the National Institute of Sciences of India 32 (B) (1966): 148-189.

Kapur A.P. 1972. The Coccinellidae (Coleoptera) of Goa. Records of the Zoological Survey of India 66: 309-320.

Klug J.C.F. 1833. Bericht über eine auf Madagascar veranstaltete Sammlung von Insecten aus der Ordnung Coleoptera. Abhandlungen der Königlichen Akademie der Wissenschaften, Berlin (18321833): 91-223.

Klug J.C.F. 1835. In: Erman, A.: Reise um die Erde durch Nord-Asien und die beiden Oceane in den Jahren 1828-30: 27-50. Naturhistorischer Atlas. Berlin.

Korschefsky R. 1932. Pars 120: Coccinellidae. II. In: Junk W. \& Schenkling S. (eds) Coleopterorum Catalogus: 225-659. W. Junk, Berlin.

Kovář I. 2007. New nomenclatorial and taxonomic acts and comments Coccinellidae. In: Löbl I. \& Smetana A. (eds) Catalogue of Palaearctic Coleoptera: 568-631. Apollo Books, Stenstrup.

Łączyński P. \& Tomaszewska W. 2012. Chapinaria, new genus of Chilocorini for Endochilus meridionalis Sicard from Africa (Coleoptera: Coccinellidae). Annales Zoologici (Warszawa) 62 (1): 1-9. https://doi.org/10.3161/000345412X633658

Leach W.E. 1815. Entomolgy. In: Brewster D. (ed.) The Edinburgh Encyclopaedia. Volume 9: 57-172. Balfour, Edinburgh.

LeConte J.L. 1860. Report on explorations and surveys, etc. from the Mississippi River to the Pacific Ocean. No.1. Report upon Insects Collected on the Survey 9: 1-72.

Leeper J.R. 1976. A review of the Hawaiian Coccinellidae. Proceedings of the Hawaiian Entomological Society 22: 279-305.

Leng C.W. 1908. Notes on Coccinellidae. III. Journal of the New York Entomological Society 16: 33-44.

Lewis G. 1873. Notes on Japanese Coccinellidae. Entomologists'Monthly Magazine 10: 54-56.

Lewis G. 1896. On the Coccinellidae of Japan. Annals and Magazine of Natural History (6) 17: 22-41.

Li J.H., Tomaszewska W. \& Ślipiński A. 2014. Ladies in stripes: taxonomic confusion in a potential mimicry complex among Wallacean Coccinellidae (Coleoptera: Coccinellidae). Zootaxa 3900 (4): 592600. https://doi.org/10.11646/zootaxa.3900.4.9

Li W.J., Huo L.Z., Ahrens D., Ren S.X. \& Wang X.M. 2017. Renius cornutus, a new genus and species of Chilocorini from Tibet, China (Coleoptera, Coccinellidae). ZooKeys 678: 121-128.

https://doi.org/10.3897/zookeys.678.11862

Linnaeus C. 1758. Systema naturae per regna tria naturae, secundum classes, ordines, genera, species cum chatacteribus, differentiis, synonymis, locis. Holmiae: Laurentii Salvii Vol. 1 Edn 10 (reformate).

Linnaeus C. 1767. Systema Nature, per Regna Tria Naturae, secundum Classes, Ordines, Genera, Species, cum Characteribus, Differentiis, Synonymis, Locis. Tom I. Pars I. Editio Decima tertia, ad Editionem duodecimam reformatam Holmiensem. Vindobonae, Typis Ioannis Thomae nob. de Trattern.

Liu C.L. 1963. Coleoptera: Coccinellidae. Economic Entomology of China, 5. Science Press, Beijing. [In Chinese.] 
Mader L. 1954. Coccinellidae. III. Teil. In: Exploration du Parc National Albert, mission G.F. De Witte (1933-1935). Institut des Parc Naitonaux du Congo belge, Brussels.

Mader L. 1955a. Neue Coleopteren aus Fukien (China). Helotidae, Languriidae, Erotylidae, Endomychidae, Coccinellidae. Koleopteraologische Rundschau 33: 62-78.

Mader L. 1955b. Evidenz der paläarktischen Coccinelliden und ihrer Aberrationen in Wort und Bild. 2. Entomologische Arbeiten aus dem Museum G. Frey Tutzing bei München 6: 765-1035.

Magro A., Lecompte E., Magne F., Hemptinne J. \& Crouau-Roy B. 2010. Phylogeny of ladybirds (Coleoptera: Coccinellidae): are the subfamilies monophyletic? Molecular Phylogenetics and Evolution 54: 833-848. https://doi.org/10.1016/j.ympev.2009.10.022

Miwa Y. 1931. A Systematic Catalogue of Formosan Coleoptera. Report of the Department of Agriculture Government Research Institute (Taihoku) 55: 1-359.

Miyatake M. 1970. The East-Asian Coccinellid beetles preserved in the California Academy of Sciences. Tribe Chilocorini. Memoirs of the College of Agriculture, Ehime University 14 (3): 19-56.

Miyatake M. 1985. Coccinellidae collected by the Hokkaido University Expedition to Nepal Himalaya, 1968 (Coleoptera). Insecta Matsumurana (New Series) 30: 1-33.

Motschulsky V. 1853. Diagnoses de Coléoptères nouveaux, trouvés par M.M. Tatarinoff et Gasckéwitsch aux environs de Pékin. Etudes entomologiques 2: 44-51.

Mulsant M.E. 1850. Species des Coléoptères Trimères Sécuripalpes. Annales des Sciences physiques et naturelles, d'Agriculture et d'Industrie, Société nationale d'Agriculture, Lyon, deuxième série 2.

Mulsant M.E. 1853. Supplément à la Monographie des Coléoptères Trimerès Sécuripapes. Annales de la Société linnéenne de Lyon (Nouvelle Série) (2) 2: 129-333.

Nagaraja H. \& Hussainy S.U. 1967. A study of six species of Chilocorus (Coleoptera: Coccinellidae) predaceous on San Jose and other scale insects. Oriental Insects 1: 249-256.

Nakane T. 1963. New taxa. In: Nakane T., Obhayashi K., Nomura S. \& Kurosawa Y. (eds) Iocographia Insectorum Japonicum Vol. 2 (Coleptera). Hokuryukan, Tokyo. [In Japanese.]

Nakane T. \& Araki M. 1959. Entomological results from the scientific survey of the Tokara Islands. VI. Coleoptera: Coccinellidae. Scientific Reports of Kyoto Prefectural University (Natural and Living Science) 3A: 45-52.

Pang H., Pang X.F. \& Huang B.K. 2002. Coccinellidae. Insect Fauna of Fujian IV: 281-357. Fujian Science and Technology Press, Fujian.

Pang H., Ren S.X., Zeng T. \& Pang X.F. 2004. Biodiversity and their Utilization of Coccinellidae in China. Science and Technology Press of Guangdong, Guangzhou. [In Chinese.]

Pang X.F. \& Mao J.L. 1979. Coleoptera: Coccinellidae II. Economic Entomology of China, 14. Science Press, Beijing. [In Chinese.]

Poorani J. 2002. An annotated checklist of the Coccinellidae (Coleoptera) (excluding Epilachninae) of the Indian Subregion. Oriental Insects 36: 307-383.

Ren S.X., Wang X.M., Pang H., Peng Z.Q. \& Zeng T. 2009. Colored Pictorial Handbook of Ladybird Beetles in China. Science Press, Beijing. [In Chinese.]

Rossi P. 1790. Fauna Etrusca, sistens Insecta, quae in provinciis Florentina et Pisana praesertim collegit. Tomus primus. Thomae Masi \& Sociorum, Liburni.

Sasaji H. 1968a. A revision of the Formosan Coccinellidae (II) tribes Stethorini, Aspidimerini and Chilocorini (Coleoptera). Etizenia 32: 1-24. 
Sasaji H. 1968b. Phylogeny of the family Coccinellidae (Coleoptera). Etizenia 35: 1-37.

Sasaji H. 1971. Fauna Japonica. Coccinellidae (Insecta: Coleoptera). Academic Press of Japan, Tokyo.

Say T. 1835. Descriptions of new American coleopterous insects, and observations on some already described. Boston Journal of Natural History 1: 151-203.

Seago A.E., Giorgi J.A., Li J.H. \& Ślipiński A. 2011. Phylogeny, classification and evolution of ladybird beetles (Coleoptera: Coccinellidae) based on simultaneous analysis of molecular and morphological data. Molecular Phylogenetics and Evolution 60: 137-151. https://doi.org/10.1016/j.ympev.2011.03.015

Scriba L.G. 1791. Beiträge zu der Insecten-Geschichte. Zweites Heft: 66-194. Varrentrapp \& Wenner, Frankfurt.

Sicard A. 1907a. Coleoptères Coccinellidés du Japon, recueillis par MM. Harmand et Gallois. Liste et description d'espèces nouvelles. Bulletin du Museum national d'Histoire naturelle 13: 210-212.

Sicard A.1907b. Espèces nouvelles de Coccinellides d'Afrique. Annales de la Société entomologique de France 76: 412-418.

Sicard A. 1909. Révision des Coccinellides da la faune malgache. Annales de la Société entomologique de France 78: 63-165.

Sicard A. 1912. Deux Coccinellides nouveaux du Congo belge. Revue de Zoologie et de Botanique africaine 1: 411-412.

Sicard A. 1913. Notes sur quelques Coccinellides de l'Inde et de Birmanie appartenant à la collection de M. Andrews, de Londres et description d'espèces et de variétés nouvelles. Annales de la Société entomologique de France 81 (1912): 495-506.

Sicard A. 1920. Descriptions d'espèces et variétés nouvelles de Coccinellides de San Thomé. Bulletin da la Société portugaise Sciences naturelles 8: 211-214.

Silvestri F. 1909. Nuovo Coccinellide introdotto in Italia. Rivista Coleotterologica Italiana 7: 126-129.

Ślipiński A. 2007. Australian Ladybird Beetles (Coleoptera: Coccinellidae), their Biology and Classification. ABRS, Canberra.

Ślipiński A. \& Giorgi J.A. 2006. Revision of the Australian Coccinellidae (Coleoptera). Part 6. Tribe Chilocorini. Annales Zoologici (Warszawa) 56 (2): 265-304.

Ślipiński A. \& Tomaszewska W. 2010. Coccinellidae Latreille, 1802. In: Leschen R.A.B.R., Beutel G. \& Lawrence J.F. (eds) Handbook of Zoology, Vol. 2, Coleoptera: 454-472. Walter de Gruyter GmbH and Co. KG, Berlin/New York.

Smith S.G. 1959. The cytogenetic basis of speciation in Coleoptera. $10^{\text {th }}$ International Conference of Genetics 1: 444-450. Canadian Forest Service Publications, Ottawa.

Weise J. 1885. Coccinellidae, II. Auflage. Mit Berücksichtung der Arten aus dem nördlichen Asien. In: Reitter E. (ed.) Bestimmungs-Tabellen der europäischen Coleopteren. II. Heft. H. Busing, Modling.

Weise J. 1887. Neue sibirische Chrysomeliden und Cocinelliden nebst Bemerkungen über früher beschriebene Arten. Archiv für Naturgeschichte 53:164-214.

Weise J. 1888. Ueber Coccinelle aus Afrika, hauptsächlich von Herrn Major v. Mechow gesammelt. Deutsche Entomologische Zeitschrift 32: 81-96.

Weise J. 1891. Neue Coccinelliden. Deutsche Entomologische Zeitschrift [1891]: 282-288.

Weise J. 1895a. Neue Coccinelliden, sowie Bemerkungen zu bekannten Arten. Annales de la Société entomologique de Belgique 39: 120-146. 
Weise J. 1895b. Insectes du Bengale. $36^{\text {ième }}$ mémoire, Coccinellidae. Annales de la Société entomologique de Belgique 39: 151-157.

Weise J. 1897. Coccinellen aus Ostafrika (Usambara). Deutsche Entomologische Zeitschrift 289-304.

Weise J. 1898a. Coccinelliden aus Kamerun. Deutsche Entomologische Zeitschrift 97-125.

Weise J. 1898b. Üeber bekannte und neue Coccinelliden. Archiv für Naturgeschichte 64 (1): 225-238.

Weise J. 1899. Coccinelliden aus Deutsch-Ostafrika. Archiv für Naturgeschichte 65: 49-70.

Weise J. 1900. Kurze Mittheilungen über Ostafrikanische Coccinelliden und Beschreibungen neuer Arten. Deutsche Entomologische Zeitschrift 113-131.

Weise J. 1902. Coccinelliden aus der Sammlung des ungarischen National Museums. Természetrajzi Füzetek 25: 489-520.

Weise J. 1905. Chilocorus reinecki [n.sp.]. Deutsche Entomologische Zeitschrift 140.

Weise J. 1906. Coccinelliden aus Madagaskar. Deutsche Entomologische Zeitschrift 203-208.

Weise J. 1910. Chrysomeliden und Coccinelliden. Verhandlungen des Naturforschenden Vereins in Brünn 48: 25-53.

Weise J. 1912. Über Hispinen und Coccinelliden. Archiv für Naturgeschichte A 78(2): 100-120.

Weise J. 1913. Coccinelliden aus Westafrika. Bollettino del Laboratorio di Zoologia General e Agraria di Portici 7: 221-249.

Yu G.Y. 2011. The Coccinellidae of Taiwan. Chemical Industry Press, Beijing. [In Chinese with English summary.]

Yu G.Y., Pang H. \& Pang X.F. 1993. Coccinellidae collected from Chebaling National Nature Reserve. In: Xu Y.Q. (ed.) Collected papers for investigation in National Chebaling Nature Reserve: 467-511. Science and Technology Press of Guangdong, Guangzhou. [In Chinese.]

Zaslavskij V.A. 1962. Novyy palearkticheskiy vid Chilocorus (Coleoptera, Coccinellidae). Entomologicheskoe Obozrenie 41: 398-401.

Manuscript received: 28 October 2017

Manuscript accepted: 11 June 2018

Published on: 23 October 2018

Topic editor: Gavin Broad

Section editor: Max Barclay

Desk editor: Kristiaan Hoedemakers

Printed versions of all papers are also deposited in the libraries of the institutes that are members of the EJT consortium: Muséum national d'Histoire naturelle, Paris, France; Botanic Garden Meise, Belgium; Royal Museum for Central Africa, Tervuren, Belgium; Natural History Museum, London, United Kingdom; Royal Belgian Institute of Natural Sciences, Brussels, Belgium; Natural History Museum of Denmark, Copenhagen, Denmark; Naturalis Biodiversity Center, Leiden, the Netherlands; Museo Nacional de Ciencias Naturales-CSIC, Madrid, Spain; Real Jardín Botánico de Madrid CSIC, Spain; Zoological Research Museum Alexander Koenig, Bonn, Germany. 